\title{
The Cerebral Cortex Realizes a Universal Probabilistic Model of Computation in Complex Hilbert Spaces
}

\author{
C. Jones
}

A model of computation describes how units of information and an organizational architecture transform input to output. In the cerebral cortex the unit of information is a normalized collection of neuronal membrane potentials representing a vector of complex amplitudes. The organizational architecture of the cortex allows for programming operator matrices in dendritic arborizations on vectors of complex amplitudes. Three well-documented facts are sufficient to define the cortical model of computation: first, cortical dendritic inputs to compute membrane potentials are, in general, complex numbers (amplitudes) that may be configured as state vectors in complex Hilbert spaces; second, normalization is a canonical neural computation, specifically, for vectors of cortical membrane amplitudes representing orthogonal states; and, third, dendritic arborizations are programmable devices in a universal sense for mathematical and logical functions. These three facts, well studied and accepted for years, are all that is needed for a simple, well-defined, and well-known model of computation. A great deal of research is presently devoted to understanding particular neural algorithms, circuits, and programming. Understanding the algorithm that a particular circuit implements is not to be confused with identifying the much more basic underlying model of computation. The analogy for classical computing is that the classical model of computing stores and manipulates information fundamentally in the form of bits essentially a finite set of possible states, typically, $\{0,1\}$. The primitive classical container of information is analogous to a two-state switch. Nevertheless, sophisticated computational circuitry and algorithms are developed on that simple classical realization. In contrast to the finite set of states found in the fundamental unit of classical information, the fundamental units of information in the cerebral cortex form a model of computation over the complex field in normalized sets of mutually inhibitory amplitudes. This means that the cortex represents information in a fundamental container of information (a vector of amplitudes in membrane potentials) that can encode discrete states over a continuum of values (no doubt down to some constant.) A well-known result from probability theory and standard neuroimaging analysis argue that these mutually inhibitory fundamental units of information may normalize complex amplitudes under the 2-norm. Sufficiently sophisticated computational means are available in the cortex to program unitary operators, state reduction and expansion operators and tensor products that operate on vectors of normalized membrane amplitudes. This model of computation is typically viewed as a probabilistic or predictive model of computation. Again, the model of computation is not a particular circuit or implementation of any one algorithm. The model of computation is the arena in which cortical circuits and algorithms are implemented. The fundamental arena then for cortical computations is a field of complex amplitudes normalized for probabilistic computation.

\section{Outline}

Part 0 : Overview

Part 1 : The Cortical Arena for Computation

Part 2 : The Hardware Device

Part 3 : The Computational Model

Part 4 : Classical Computing with Levers, Marbles, Crabs and $1.4 \mathrm{~nm}$ Die Shrink

Part 5: A Sketch of the Logical Primitive

Part 6: What the Computational Model Looks Like in the Representation of Spacetime in the Entorhinal Cortex and for Bosons in an Optical Lattice

Part 7 : Podcast Interview with J von Neumann and N Bohr, Cognitive Neuroscientists

Part 8 : The Comic Strip Version 


\section{Part 0 : Overview}

Part One gives a very quick and dirty sketch of the "arena" for computation in the cerebral cortex.

Part Two goes into just enough detail to describe the substrate for the cortical model of computation.

The goal is to give a simple overview of the basic computational devices in the cortex. The analogy would be an overview of the basic devices in a classical microchip - transistors, logic gates, etc. - making possible a functional understanding of the computational model at work without requiring expert knowledge of semiconductor devices. The essential hardware devices of the cortex are clearly present and have been well-described by neuroscientists for some time - decades in many cases. A functionally sufficient understanding of the computational primitive, both in the classical model and in the cortical model, is simple and accessible.

Part Three describes how the cortical devices overviewed in Part Two satisfy as logical primitives and operators in the model of computation. This is taken more slowly so that careful attention can be given to what is required for this model of computation as stated in broadly accepted postulates from canonical texts in the field. The requirements for this model are quite minimal and clearly present in the cortex. There are no spatial or temporal scale requirements in the postulates for this model of computation. The postulates of the model only specify a mathematical and logical architecture that must be present. The reader is strongly encouraged to actually study the postulates of the model for themselves in the canonical texts and not to unquestioningly accept being told the model is "about atoms and physics."

Part Four considers four extremely different realizations of the classical (binary) model of computation. The hope is to give the reader an appreciation for how radically different systems can be in scale or types of devices and yet still be the same fundamental model of computation. A model of computation is a very, very general abstraction that is independent of any particular realization. This is not to claim that principles of physics will not prevent some proposed realizations. But, it is simply wrong to rule out the possibility of certain physical realizations in the absence of physical principles preventing the realization. Therefore, the sober and conservative procedure for identifying and confirming the model of computation for a given physical device requires establishing that it satisfies completely the definition of a model of computation. This paper will describe how the cortical model of computation satisfies the postulates for a well studied universal ${ }^{1}$ model of computation as the postulates are given in the canonical text for the academic field. Along the way, we will point towards some of the other possible ways that the same model is defined or interpreted.

Part Five is a nice picture of the logical primitive in the cortex from the neuroscience literature.

Part Six gives a couple of examples of how the fundamental nature of the model gives rise to identical structures seen in the entorhinal cortex and between bosons in an optical lattice.

Part Seven is a podcast interview with the cognitive neuroscientists $\mathbf{J}$ von Neumann and $\mathrm{N}$ Bohr.

Part Eight is the comic strip version of this paper that will save you some time. ${ }^{2}$

\footnotetext{
${ }^{1}$ More below on what is meant by a "universal" computer. But, essentially, it is a programmable, general computing device that is capable of modifying itself. This means it is not "trivially" a computer in the way that maybe a flower is a computer because it can compute only ... how to be a flower ... you can't run an email server or do word processing on it.

${ }^{2}$ A draft of this paper titled The Cortical Model of Universal Computation circulated by email in 2014 to a variety of physicists, neuroscientists, cognitive scientists, computational complexity theorists, and others, in an attempt (by a confused computer engineer) to find someone to explain why many seem to be under the impression that the model of computation in the cortex is classical and/or why it is not the model described in this paper. The answers tended to be a variety of "You're a crackpot", or "You just don't understand how complicated it really is", or, for those somewhat sympathetic, "Yes, but you have to give 'empirical' evidence." If the interviewee was presented with the evidence that the cortical model does appear to satisfy the canonical postulates of the model the response tended to be "Well, those postulates are really just simplifications. That's not how it really is." or "Decoherence doesn't allow for that" when decoherence is not in the postulates... So, either the only way to understand this model is by some kind of magical transference into enlightened "knowing" ... or there is a comprehensible definition in the postulates that may be applied to answer the question. This author has been somewhat taken aback at the oftentimes hostile reaction to this thesis when the core of the argument simply is that the cortex realizes a general kind of probabilistic model of computation.
} 
"Science is belief in the ignorance of experts."

(Feynman, 1969)

"The first principle is that you must not fool yourself - and you are the easiest person to fool. " (Feynman, 1974).

"If you've made up your mind to test a theory, or you want to explain some idea, you should always decide to publish it whichever way it comes out."

(Feynman, 1974).

\section{Part 1: The Cortical Arena for Computation}

A model of computation is an arena in which realization (programming) of algorithms may occur. ${ }^{3}$ Describing a computational model typically begins by defining or identifying the fundamental logical unit that will store information for processing. Next should be given the general rules for how logical and mathematical operations are carried out with those primitive logical units to transform input into output. There can be extreme variation in the physical realizations of computational models and the applications that are programmed using physical hardware. A model of computation is like a translation layer between the software of mathematical and logical algorithms and hardware device architectures. It is a statement of the necessary and sufficient functioning of the hardware for computing.

The consensus fundamental unit of information in the cerebral cortex has been the membrane amplitude or potential. The cortex uses this information, in general, for predictive or probabilistic computation of outputs. For decades neuroscientists have understood that representation of information in the cortex by the membrane potential is in the form of a complex number. In this model, positive or negative amplitudes and phases can store and compute meaningful information. These amplitudes are used by the cortex in dendritic computations as positive or negative inputs to dendritic arborizations that encode mathematical and logical functions. This paper will argue that the fundamental logical primitive of the cortex is not the single membrane amplitude but is a normalized set (a vector) of complex amplitudes in mutually inhibitory columns or groups. Such an information primitive may be used for a probabilistic model of computation.

So, what kind of model of computation is found by implementing a probabilistic model of computation with positive and negative complex numbers? A negative number underlying a probability, while it may sound strange at first, can be understood in terms of representing evidence against an outcome or as the negative of "being", like anti-support or simply as negative weight for an output. The usual term is interference. Due to normalization the negative numbers never appear as the actual probabilities but only serve as a means for interference to exist between possible states.

The probabilities are encoded as synaptic excitation (increases the probability) or inhibition (decreases the probability). The set of amplitudes related to the state of a system can be represented as a vector. This is a vector in a complex Hilbert space because cortical operations easily allow for an inner product between vectors and the various means for encoding of amplitudes realizes a practically complete metric space. For a probabilistic model this vector must be normalized and indeed normalization is believed to be a canonical operation throughout the cortex. Theoretical implications from computational theory and experimental evidence from neuroimaging studies of functional connectivity suggest that the 2-norm is likely present as a normalization. If operators programmed in dendrites map unit vectors in the 2-norm to other unit vectors under the 2-norm then these are unitary operators. This model allows predictive paths of operators to interfere constructively and destructively in a way that is different from the classical model of (probabilistic) computation because in the classical model the numbers underlying probabilities are not negative.

\footnotetext{
${ }^{3}$ This section is a blatant imitation of Scott Aaronson's lecture on how to demythologize this model of computation. This model is not about "matter, or energy, or waves, or particles" (Aaronson, 2006b).
} 


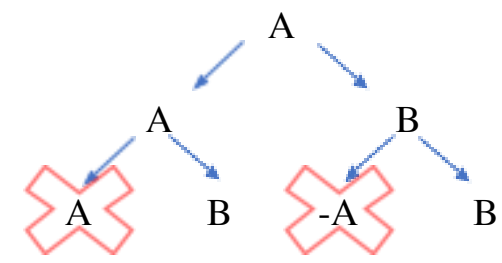

Figure 0. Computing with fundamental units that may hold positive or negative amplitudes exhibits non-classical interference of the fundamental units of information.

If the model by such interference produces only amplitude in a single possible outcome then that will be a computation said to measure or observe that outcome. In that case, the cortex will have computed that it is observing or has measured that outcome. If the normalized amplitudes are distributed out in possible outcome states then that will be a computation that has not measured or observed a single outcome. In that case, the cortex has not computed that a single outcome has been measured or observed. This model of computation obviously contains the models of classical probabilistic computation and classical deterministic computation as subclasses. Thus, it is possible for the cortex to also realize algorithms or circuits that are purely classical in either of those models as well. Indeed, if information classified as resulting from observation or measurement will not involve negative amplitudes then any algebra over that information in the cortex will be a classical logic. However, it will always be possible to construct different classical algebras over the underlying non-classical structure .

And that is all there is to it.

\section{Part 2: The Hardware Device}

The cortex unfolded reveals a computational sheet roughly a square meter wide and $2.5 \mathrm{~mm}$ thick with several notable features (Merker, 2007). Different sections may be encoded with the circuitry for specialized functions over various input-output domains (Douglas and Martin, 1991; Braitenburg and Schüz, 1998; Shipp, 2007). Although details are under debate there is compelling evidence that the neurons of the cortical sheet cooperate to some extent in functional units, groups or columns (Mountcastle, 1997; Hosoya, 2019). A first thing to note is that there is a parallel recurrent group architecture among the columns that allows for robust fault tolerance (Koch, 1999) - made possible by what is called the threshold theorem and that is typical of computational structures (von Neumann, 1956; Nielsen and Chuang, 2000).

This paper will use the terminology of the cortical column, macrocolumns and minicolumns (or microcolumns), for the specialized functional units, groups or modules found across the cortex. ${ }^{5}$ The cortical macrocolumn is a group of cells forming a functional set perpendicular to the cortical surface. Each macrocolumn is itself composed of an estimated 50 to 100 minicolumns with each minicolumn containing 80-120 neurons, except in the visual cortex where there can be twice that number

\footnotetext{
${ }^{4}$ The classical probabilistic model of computation, which goes by BPP, is contained in the model considered here, the "non-classical" complex-Hilbert BQP. Although there is evidence, it is not proven that these models of computation are not equal (Aaronson, 2009). The two logics are metastructures for dealing with different algebras, lattices, as their models. Logic is not uniquely empirical since it can simultaneously describe distinct realities. The propositions of the non-classical logic correspond to elements of a Hilbert lattice and are not directly linked to measurement values. Such logic employs models which evaluate particular combinations of propositions by mapping from a set of propositions to an algebra (lattice), through which a correspondence with measurement values indirectly emerges. Since the algebra must be an orthomodular lattice and cannot be a Boolean algebra then this non-classical logic which has an orthomodular lattice as one of its models is "empirical" whenever describing non-classical measurements, simply because it can be linked to its algebraic model which serves for such a description: an orthomodular Hilbert lattice, that is, the lattice of closed subspaces of a complex Hilbert space (Pavičić, 2016). ${ }^{5}$ The terminology in the neuroscience literature includes at least columns, bundles, groups, COUs, etc. (Innocenti and Vercelli, 2010, for example.) These kinds of distinctions are distinctions about circuit or architecture design that are above the much more fundamental notion of the model of computation. The underlying model of computation (classical or non-classical) is the arena in which simple or sophisticated circuits may be programmed.
} 
(Buxhoeveden and Casanova, 2002b). This same basic organization is evident throughout the cortex with an estimated $2 \times 10^{8}$ minicolumns or $2 \times 10^{6}$ macrocolumns. Functionally, the neurons in a minicolumn encode a common feature or aspect of some information source with neighboring minicolumns encoding related aspects. The specific circuitry of a column is not what is important here but that these columns cooperate in some computational way to represent the possible interpretations of input (Tsunoda et al., 2001; Douglas and Martin, 2004; Cain et al., 2016). Within this set of cooperating minicolumns is the complete (mutually exclusive and exhaustive) representation of some specific input source. The domain of the input could be anything at all that gives the cortex input, input from another part of the cortex, or recurrent input from the columns themselves.

What can be said about the computational properties of a group of cells such as the minicolumn considered as a physical computing device? Given a population of cells with identical threshold distributions, if all receive the same input then it was observed that an output firing rate can be defined for the unit (Knight, 1972). The threshold for firing, generating axonal spikes, is not fixed but is some probability distribution so that every time a spike is generated the threshold is set to a new value drawn from the probability distribution (Gabbiani and Koch, 1998). Important for our general viewpoint, it has been shown that for such an ensemble of cells the relationship between the input and the output firing rate is a linear one (Gestri, 1971; Cain et al., 2016). Koch (1999) expounds on this remarkable maintenance of linearity for neuronal functions, saying, "despite all the nonlinear mechanisms known to exist in the nervous system, evolution conspires to construct neurons that act in a perfectly linear manner". This collection of fundamental units of information, this cooperating set of logical primitives, a firing rate ensemble of cells, operates linearly. This property ensures insensitivity to small errors and is a feature shared by discrete and probabilistic models of computation but not by analog or continuous models (Aaronson, 2013). This gives the first key feature of the cortical model of computation :

\section{Cortical neurons are linear operators.}

The output of the minicolumn's firing rate neurons are typically modeled by a charging RC element and passing the resulting membrane potential through a stationary nonlinearity, implemented by an ideal op-amp (Arbib, 1995). Interacting firing cells in a network are coupled through synaptic connections that have a scalar weight, $W_{i j}$, for presynaptic neuron $i$ and postsynaptic neuron $j$ (Bernander, 1993; Bernander et al., 1994; Zador and Dobrunz, 1997). For such a network it is possible to list these variable inputs in connectivity matrices (Koch, 1999).

The minicolumn's volume is taken up mostly by the dendritic arbors of the cells, as is the cortex itself (Segev, 2006). Dendrites make up 98\% of the cell's surface area. The dendrites are where the cortex computes (Mel et al., 1998; London and Segev, 2004; Branco and Hausser, 2010; Papoutsi et al., 2014). Koch (1999) emphasizes that these are nontraditional computational devices and calls the common perception of them as threshold sum units "moronic". The actual operation is based upon their morphology and the synaptic input (Koch, 1999; Douglas and Martin, 2004). The input synaptic weights to the neuron's dendrite introduce a voltage attenuation between the synaptic site and the soma. In general, this is a complex number (Rall and Rinzel, 1973). For a general complex number input both the amplitude and phase parts are available for encoding information (Fries et al., 2007; Młynarski, 2014; Młynarski, 2015). Synaptic weights in the cortex are complex amplitudes.

\footnotetext{
${ }^{6}$ This is inclusive of rate and temporal coding. Koch (1999) prefers the term correlation population code. Note how important linearity of the neuron is for this model. All of the following discussion of the superposition of complex numbers in the model of computation depends on this fact. For reflection on this "deep principle" in neural function see Koch (1999).

${ }^{7}$ Complex numbers deliver a unique analytical structure being algebraically closed, having well-defined division and complex differentiability. Neural encoding of complex-valued associative structures may offer advantages (Aizenberg, 2011; Masuyama, 2015).
} 
Synapses encode information for some receptive field in the form of complex numbers. The actual complex number value depends upon the membrane potential but also the axonal and dendritic morphologies that connect the membrane potential to other neurons (or recurrently to itself.) The membrane potential, like any measurement, is meaningless, in itself, without projection onto some measurement basis (Derrida, 1967). The dendrites span the neuron's oscillatory cycle with branching allowing for an operation of positive or negative complex amplitudes to be applied up and down the phases of the wave function as input to the operation programmed by the morphology. Any such input may be formalized in matrices (Hines, 1984; Koch, 1999) Thus, the membrane potential is a representation of information by complex numbers as a result of matrix inputs of complex numbers:

\section{The cortex encodes information in complex numbers.}

The minicolumn cells' membrane potential can be the result of many different logical or mathematical operations encoded in dendritic morphology. A substantial body of evidence supports not only addition and subtraction but multiplication, division, and correlation-like operations (Koch and Poggio, 1992). Also available are polynomial functions, so-called second order synapses, such that connectivity matrices specify the function by specific spatial dendritic relationships (Bruck, 1990; Bruck and Smolensky, 1992; Mel, 1992; Mel, 1993; Mel, 1994). Boolean functions are available such as the exclusive-or, XOR, which may be generalized as a controlled-not, CNOT (Fromherz and Gaede, 1993).

Cortical neurons can implement linear phase shift or delay operations on input. The physical mechanisms available include the morphoelectrotonic transform of the cell and moving the synaptic input site within the dendritic morphology. (Agmon-Snir and Segev, 1993; Zador et al., 1995). A linear operation that involves the multiplication of two outputs, one temporally delayed is possible (Reichardt, 1961). The capacity of the fundamental cortical unit of information to encode relative phases will be important in the following consideration of the computational model.

Recapping what is known from the above neuroscience literature:

The cortical sheet has a ubiquitous structure of functional units or columns. Each macrocolumn is composed of minicolumn clusters of neurons. These minicolumns as a group can act as a computational primitive representing orthogonal features or aspects of some source probabilistically by taking complex number input matrices and performing a linear operation that is represented in the membrane potentials as complex amplitudes. These physical architectures implement logical and mathematical functions, especially multiplication and phase shift operations, on matrices of complex numbers. Establishing a model of computation should be based upon clear physical evidence of the operation of the devices.

Remember, this overview is intended to be sufficient only to explain the computational model and not any particular realization of a program or circuit. The cortical columns, like the semiconductor layers on a microchip, may have highly sophisticated layout and circuitry that is tied into other parts of the computational substrate in extremely nontrivial ways. Indeed, there may be differentiated Hilbert spaces defined "within" the vertical layers of the cortical sheet, between different columns, or groups shared among spaces. But those details are not important for the purposes of this thesis. The question here is very fundamental in looking for the computational primitive of information like the transistor/bit primitive information machine in integrated circuits. If such a primitive of information is identified, if common logical or mathematical functions can be used, if input and output means are present, and if the devices are somewhat fault tolerant, then there is a feasible model of computation.

\section{Part 3: The Computational Model}

The groups of membrane potentials are the result of varying amplitudes in dendritic branches (Douglas and Martin, 2004). This is the physical device upon which the operations listed above occur. These varying amplitudes include positive and negative changes to the resting potential due to two types 
of synaptic input: excitatory and inhibitory (Miles, 2000; Thompson et al., 2002; Contreras, 2004). The minicolumn unit of these excitatory pyramidal cells and inhibitory interneurons forms a naturally oscillating waveform that encodes the state of some system (Ward, 2003; Fries et al., 2007; Fries, 2009; Suppes et al., 2012). An oscillatory system can be formalized in terms of complex numbers, amplitude-phase, with the exponential-sinusoidal relationship. Thus, the computational primitive, through the specific circuitry of the synaptic connections and dendritic arborizations, encodes a wave function. The phase part of the function gives it a "wavelike" character (Penrose, 2004). While the oscillation of the membrane potential is local it may be part of global, cortex-wide traveling waves (Lubenov and Siapas, 2009; Muller et al., 2018).

Each cortical minicolumn can encode for a particular state, feature, or aspect of a source stimulus. The encoding is through the function, such as those listed above, being wired or programmed by the combination of dendritic morphology, synaptic weights, and their modulation. The source here is simply something, some system that results in the attenuation appearing in the postsynaptic dendrites of the column. Like any information processing system, the cortex can be analyzed at several levels. This can seem almost overwhelmingly so for the cortex (Koch, 1999). Discrete minicolumn spaces are the best candidates for the fundamental logical primitive - a computationally meaningful level of representation even if the terminology is under debate and the possible ways the columns may operate are not fully understood (Defelipe, 2012). So long as a minicolumn can function as one of a set of orthogonal input states with membrane potentials encoding the complex amplitudes for that state (possibly many different amplitudes relative to different bases), connective morphologies encoding logical and mathematical functions, and some degree of fault tolerance then this model will work.

The minicolumns are found as a coherent functional set within a macrocolum or "macrogroup". The macrocolumn expresses the full set of values for any receptive field of input it operates over. Its minicolumns cooperate to explore all possible interpretations of receptive field input and select consistent interpretations (Douglas and Martin, 2004; Douglas and Martin, 2007; Tsunoda et al., 2001). Is there a known mathematical formalism that seems appropriate? A probabilistic model is suggested because all outcomes are accounted for and encoded within the receptive field by the minicolumns and the device acts in a discrete, linear, probabilistic fashion. The macrocolumn then can act as a complete measurement, meaning no further refinement for the response (Peres, 1993). The completeness condition is satisfied by the physical architecture in that all responses are accounted for. Subsequent responses may change the probability distribution but that can't be known until it arrives. The minicolumns form an informationally complete set over their receptive fields. This probabilistic mathematical model on cortical hardware is described as an information system for predictive encoding (Adams et al., 2013; Cisek, 2007). However, these probabilities do not represent ignorance in the way probabilities do in classical probability theory. The cortical amplitudes are a record of intrinsically random events (Bub, 2016) or sui generis aspects of what constitutes the cortex's universe of information (von Neumann, 1962).

\footnotetext{
${ }^{8}$ The normed division algebras, by generating the exceptional Lie Groups, are argued to provide the computational arena for physical theories (Wigner, 1960; Witten, 1998; Baez, 2002; Penrose, 2004; Stewart, 2008). This is only an arena - meaning, there are no predetermined operators (Hamiltonians) for determining the model of an experience. The arena is just the nature of the "design space" within which the operators may be determined. Thus, this model of computation requires experimentation (Tavakoli and Gisin, 2020). This may be called learning. The learning (programming) may be done through evolution or in real time by the computational device - a posteriori. The device would, of course, be in possession of the logical "geometries" of its model a priori (Euclid, 300 BC; Kant, 1781; Pitowsky, 2008b).

${ }^{9}$ The function of what is known as a Markov blanket is given "for free" with the generic assumptions of this model of computation (Fields and Marciano, 2019). This non-classical model appears to be a better model for some cognitive data from studies of human decision making (Busemeyer et al., 2020). The model may reduce "surprise", prediction-error signals, by overlaying neural amplitudes with Boolean algebras - a classical logic or "story about the world." These are always perspectival projections onto the amplitudes in the cortical spaces and not an independent, external global "logic" or Boolean algebra. The amplitude is then no longer "surprising" only in the context of the model. It is always possible to "look afresh" at the "same" amplitude and see it detached from a contingent model. The dissolution or deconstruction of a Boolean algebra over a set of amplitudes has been a common theme in religious and philosophical activity (You want references for that kind of statement? Very well, start with Rorty, 1989). Any classical Boolean story about the world can only ever be valid up to a point. The global
} 
The key in this physical realization of a probabilistic model is the mutually inhibitory nature of coupled sets of minicolumns or groups of cells (Ben-Yishai et al., 1995; Brunel, 2000). This inhibition allows for normalization among the coupled minicolumn's cells that is proportional to their excitatory amplitudes (Bloomfield, 1974; Torre and Poggio, 1978). There is evidence that normalization is a canonical neural computation - by analogy to normalizing a vector, as described by Carandini and Heeger (Carandini and Heeger, 2013). Together with completeness this allows the network of cells to maintain a normalization condition, meaning that all discrete minicolumn amplitudes sum to 1 , or a $100 \%$ representation of response for a coherent functional set.

If cortical information is represented in mutually inhibitory, orthogonal minicolumns that each encode complex amplitudes, then what is the formal normalization condition? Fortunately, there is a result from Gleason (1957) which states that in general for probability spaces with the structure found here the measure that works is that the sum of the squares of the magnitudes of the complex numbers must be equal to 1 . This means that negative complex numbers in the model (inputs as dendritic inhibition) can appear as a form of interference between states while the actual realized probabilities are always positive.

That is, for $N$ amplitudes that represent some system in $N$ minicolumns (encoded in the amplitudes of membrane potentials) the normalization condition maintains

$$
\sum_{i}^{N}\left|a_{i}\right|^{2}=1
$$

where $a \in C$ and $|a|=a \cdot a^{*}$. This should be understood as a statement about the oscillatory or "wavelike" encoding of information by the logical primitive. Given a complex number basis as the measure then the structure naturally imposes the normalization condition. The oscillatory waveform imposed by the interneurons connecting the mutually inhibitory columns maintains the 2-norm between the membrane potentials. The Pythagorean identity (unit circle or sphere) is a simple picture of this but Gleason (1957) gives the general measure for any such space. ${ }^{10}$ An important feature is that the square of the amplitude of the complex traveling wave is a constant - a constant probability measure independent of the variables of the wave function!

It is important to see evidence for the 2-norm in the correlations of neural operations. Neuroimaging analysis confirms the 2-norm can meaningfully measure the functional connectivity or correlations between neurophysiological events (Friston, 1997). Friston says, "The vector 2-norm can be used to measure the degree to which a particular pattern of brain activity contributes to a covariance..." Correlations are normalized covariances. It is not argued here that the 2-norm is the only possible expression of cortical activity - indeed information can be estimated with the 1-norm, weighted norms, or mixed norms. The neural architecture can allow for correlations in other norms. However, if a model requires that information be assembled in vectors of amplitudes, the transitions are linear maps, and the information produces normalized probabilities for predictive computing that are preserved by the mappings then there are only three options where interesting transformations exist: deterministic models (but then vectors are not needed as the device is only ever in one of a set of permutations), classical probabilistic models (but then there is no need for negative numbers underlying the probabilities (Feynman, 1982)), or the non-classical model described here (Aaronson, 2004; Aaronson, 2013). Only the 2-norm, among those models, can be induced by an inner product. What is important for the thesis here is

structure of the (here argued cognitive) model is non-Boolean - there can be no ground in the classical sense of a fully determinate globally Boolean non-contextual assignment of values to all relevant observables (Janas et al., 2019).

${ }^{10}$ For reflection on this "2-norm" and the role of complex numbers see Aaronson (2004) and Penrose (2004). Only the 1-norm or

2-norm can give non-trivial linear transformations for this type of model. All norms in finite dimensional linear spaces are equivalent in the sense that they are within a constant factor of one another and define the same topology - an equivalence relation. However, while all norms are identical for topological purposes they are not necessarily identical for metric purposes. For example, the 2-norm and the $\infty$-norm are topologically isomorphic but not isometrically isomorphic (Sokal, 2013). 
that the 2-norm is widely found in correlations of cortical activity - there is evidence that it can and does give a meaningful measure over cortical information:

\section{Cortical correlations may form under the 2-norm.}

The vector of amplitudes is a set of mutually inhibitory minicolumns such that the set taken together represents the probabilistic state of some system - the information primitive of the cortex. These amplitudes can be thought of as forming an $n$-tuple list of complex number membrane potentials. Such a list is a complex number vector in the vector space $C^{n}$. Thus, the model forms a normed division algebra over the complex field. By the Gelfand/Mazur theorem this algebra is isomorphic only to the complex numbers (Rudin, 1973). This "state vector" of the system is naturally normalized to a unit vector by mutually inhibitory interneurons. These are the fundamental logical units of the cerebral cortex. Represent such a vector by the symbols ${ }^{12}$

Given the mathematical operators above the following function can be encoded for two vectors $\left(y_{1}, \ldots, y_{n}\right)$ and $\left(z_{1}, \ldots, z_{n}\right)$ expressed in the matrix (linear algebra) formalism (Nielsen and Chuang, 2000)

$$
\langle Y \mid Z\rangle=\sum_{i} y_{i}^{*} z=\left[y_{1}^{*} \ldots y_{n}^{*}\right]\left[z_{1} ; \vdots ; z_{n}\right]=a \quad \text { where } \quad a \in C
$$

The order of arguments for this function makes a difference, $\langle Y \mid Z\rangle=\langle Z \mid Y\rangle^{*}$. It is also linear in the second argument, by linearity in the neurons and operators as described above. And $\langle Y \mid Y\rangle=0$ if and only if $|Y\rangle=0$. Such a function that takes as input two vectors from a vector space, produces a complex number, and satisfies the above requirements is an inner product. This inner product can be seen as a measure of similarity between two vectors in the model (Busemeyer and Bruza, 2012). Orthogonal vectors are defined by the condition that $\langle Y \mid Z\rangle=\langle Z \mid Y\rangle=0$. A norm or length for a vector $\|Z\|=\sqrt{\langle Z \mid Z\rangle}$. The inner product of a vector with itself equals the squared length $\|Z\|^{2}=\langle Z \mid Z\rangle=Z^{\dagger} \cdot Z$ where $Z^{\dagger}$ is the adjoint operation. This finite dimensional complex number vector space with inner product is called a Hilbert space ${ }^{13}$ (Horn and Johnson, 1985). Mel $(1992,1993)$ shows how this function may be implemented in neuronal dendrites with multiplication as a similarity detector. The 2-norm is the only $p$-norm $(1 \leq p<\infty)$ that comes from an inner product.

Products such as this inner product can be considered as learning or programming rules for synaptic potentiation (Hebb, 1949). In the neuroscience literature this is usually expressed as $\Delta W_{i j}=V_{i} V_{j}$ where $\Delta W_{i j}$ is the amplitude of the synaptic coupling between presynaptic neuron ${ }_{i}$ and postsynaptic cell $j$; $V_{i}$ and $V_{j}$ represent some "activity" of these neurons. There are variations on the rule but a key observation is that it is asymmetric (generally noncommutative) with the presynaptic input preceding

\footnotetext{
${ }^{11}$ Vectors are very general entities (Maudlin, 2011). Notation is borrowed from the natural philosophers who have been using this model of computation for some time now.

12 This paper uses the hieroglyphics of physics while acknowledging that the model is not "owned" by physics. With a Rosetta stone it is possible to move between the frameworks of physics, logic, category theory, topology, and computer science - objects and morphisms between objects (Baez and Stay, 2009). In this direction see the work of Girard (2011) on linear logic as a way of handling information that cannot be arbitrarily copied or deleted (no-cloning and no-deleting) - the logic of tensor products on Hilbert spaces.

${ }^{13}$ The Hilbert space formalism is best understood as a general framework for handling probabilities in which only some observables can take on definite values (Janas et al., 2019). The Hilbert space formalism can be derived from the fact that any state can be decomposed into a convex mixture of discriminable states (Barnum et al., 2014).
} 
activity in the postsynaptic cell for the function. This can be a measure of coherence between two vectors in the model.

There are two possible cases following activity at a synapse:

(1) The synapse sets an amplitude in the dendrite and the synapse does not change its programmed weight (amplitude), or

(2) The synapse sets an amplitude in the dendrite and also changes its programmed weight (amplitude) in what is called synaptic depression or enhancement. (Remember encoding complex numbers can mean changes in the magnitude or phase of the information.)

The first case of firing (or not) with no change in synaptic weight is identified below as unitary (linear) evolution. The second case of a synaptic change is identified as a discontinuous jump in the state vector. Understanding of synaptic plasticity is not complete. It can occur on many different time scales as a form of memory in the computational model and, of course, there is much to say about the biophysics of what is happening at the synapse (Koch, 1999). But for the purposes here it is possible to stop before going into deeper biophysical details and be satisfied with the knowledge that the synapse is a programmable amplitude (complex number) input to the dendrite.

To summarize in the model's first postulate (following Nielsen and Chuang, 2000).

Postulate 1: The cortical macrocolumn of mutually inhibitory minicolumns over receptive fields can realize a vector state space of orthogonal states with an inner product. Probabilistic or predictive information about the state of the input to the receptive field can be encoded in normalized state vectors with complex amplitudes.

Notice what this does not specify: the dimension (number of minicolumns) of the state space or the details of the state vector (the amplitudes of the membranes)! The cortex can only establish these through the network and memory mechanisms that set synaptic connections and weights. This "wiring up" process could be called programming or learning and could result from a genetic (pre-installed) code or the environmental experiences of the cortex. This postulate only describes the arena in which such programming may occur. This is an important point because this paper is not considering specific realizations of cortical circuitry but the primitive model that underlies all cortical circuitry. Just as with classical circuits, the actual cortical circuits can be wildly different in appearance and function, implementing things as different as mapping space in the entorhinal cortex ${ }^{14}$ or color perception (Berthier

\footnotetext{
${ }^{14}$ The cortical model of computation produces a simulation we call space as a superposition (the positive and negative interference) of wavefunctions (Lloyd, 2006; Moser and Moser, 2008). Positive and negative amplitudes where the former reinforces activity and the latter suppresses or negates it is a common underlying model for triangular lattice pattern generation (Turing, 1952; Rowland et al., 2016). A way of saying this is that the model supports generation of classical-type spaces for "on-shell" amplitude models. The general notion is essentially construction of a convex polytope (the geometry of possible Boolean algebras) generalizing the simplex in projective space (combinatorics of the hypersimplex) (Penrose, 2004; Pitowsky, 2008b; Bub, 2016; Arkani-Hamed and Trnka, 2014; Schmid et al., 2019). The amplitudes are the building blocks (volumes) from which are programmed amplituhedron facets. Classical (logic) models and non-classical models emerge. The facet logics are the possible amplitude worlds (evidenced in the Rashomon effect of theories). But the point is that the representation in the model called space (or spacetime) is not fundamental but functions as a simulation within the cortical model - thus not fundamental to the model but a programmed construction from the model. The "discoverable" mathematical "physics" of spacetime is thus a consequence of how spacetime is fundamentally programmed by the model - the mathematics and "laws" all coming from the model itself. Discovery of such laws is the discovery of the laws created through the model. The cortical model has no built-in spacetime structure - it is itself only an arena for building such structures out of amplitudes, interference, normalizations and operators. Those structures may be installed following DNA instructions or by external programming. This is model solipsism meaning, there certainly is an unforgiving reality, but knowledge of it begins and ends with representations within the model of computation of the device subject to that reality. The device can only speak about external reality by speaking about its representations of reality and ultimately those are characterized by the model of computation of the device. The cortical arena is a stage for programming predictive representations of reality. The representations are only possible ways of seeing - always fundamentally probabilistic and contextual.
} 
and Provenzi, 2019). But, just as classical computing can realize very different functional blocks, the underlying primitive physical devices are the same. For the classical computer these are binary bits in ${ }_{15}$ transistors. For the cortical computer these are mutually inhibitory amplitudes in minicolumn membranes.

What is the simplest description possible of a system within this framework? Consider a single minicolumn state space, $C^{1}$. The minicolumn's 80-120 neurons are bundled together to form a single primitive logical unit. These cells could be jittered or noisy parallel representations such that the variable is a more fault-tolerant temporal average firing rate (Koch, 1999). The variable is the complex amplitude of the minicolumn's membranes (possibly different in different bases), encoded in synaptic amplitudes, magnitudes and relative phases, among axonal and dendritic morphologies to other neurons. (To what degree subsets of a minicolumn's neurons down to even a single neuron may satisfy fault tolerance for this model is left open (Barlow, 1972; Quiroga, 2012).) Because there is no mutual inhibition through the normalization condition with other minicolumns the single stand-alone minicolumn's behavior may be interpreted as a single complex amplitude unit of information. This means that its value is some complex number, a random variable, in the interval of the unit's operational frequency. The key to note here is that due to the absence of excitatory and inhibitory coupling to other minicolumns there can be no information dependency for this variable in other measure spaces. Thus, the membrane amplitude captures all possible information for this system in this single minicolumn state space. While global phase factors can be dropped this space, $C^{1}$, can encode phase information. This can be through various codes, by varying the frequency, or making use of interspike intervals (Koch, 1999). This single unit may encode part of a representation with other column vectors.

If a state space of one minicolumn functions as a probabilistic bit over $C^{1}$, then what happens if two minicolumns are coupled together in mutual inhibition to represent a state vector? This coupling is achieved by the two mutually inhibitory amplitudes under the 2-norm. ${ }^{16}$ This requires that the respective amplitudes interfere with each other in the sense that amplitude in one minicolumn must "push down" the other minicolumn's amplitude through the action of their coupled inhibitory interneurons (Brunel, 2000). Remember, both the amplitude and the phase of the amplitudes must be considered in how the interference takes place. If the one-minicolumn state space is like one "bit" then this must be a "2-bit" state vector. But the two bits don't behave like two classical bits. Classical bits do not interfere with each other's amplitudes under normalized superposition. Since these are "cortical bits" it can be called a "2-cobit". A 2-cobit state vector then may be written as

$$
|\Psi\rangle=a_{1}|1\rangle+a_{2}|2\rangle
$$

Where $|1\rangle$ and $|2\rangle$ are simply arbitrary labels for two distinct, orthogonal, measurable outcomes or responses of the system represented by the two minicolumns with respective membrane potentials $a_{1}$ and $a_{2}$.

\footnotetext{
${ }^{15}$ A popular model of this distribution of normalized weight profiles (amplitudes) across a set of neuronal states is the continuous attractor neural network (CANN). The dynamics of a CANN are described by the wave functions of the quantum harmonic oscillator as the basis (Fung et al., 2009; Fung et al., 2015). There are varieties of CANN attractor dynamics (ring, field, etc.), but the key feature is the normalized vector of amplitudes representing states. Neuroscientists suspect this is a canonical circuit in the cortex (Wu et al., 2016).

${ }^{16}$ Norm observable operator models use a set of linear operators to update a state. They represent probabilities by the square of the norm of system states. Norm observable operator models capture all Markov chain describable processes embedding them into an inner product space (Zhao and Jaeger, 2010).

${ }^{17}$ Busemeyer and Bruza (2012) reference Blutner and Hochnadel (2010) who coined the term "cubit". Also, Moore and Mertens (2011) use the term "cobit" for "coherent bit" from Harrow (2004).
} 


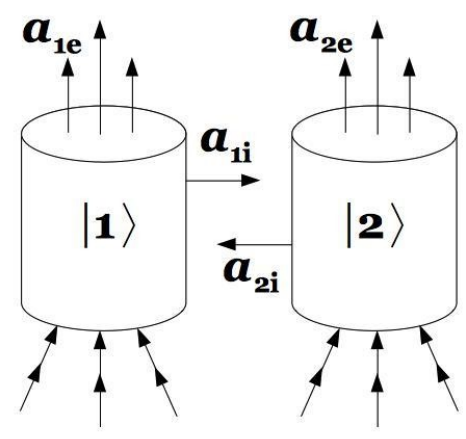

Figure 1. Cartoon for mutually inhibitory minicolumns forming a 2-cobit logical primitive with excitatory and inhibitory amplitudes. Note inhibitory connections are to basal dendrites. Recurrent connections from axonal projections back to basal dendrites not shown.

Then express a general $n$-dimensional state vector, with $n$ minicolumns, as an $n$-cobit

$$
|\Psi\rangle=a_{1}|1\rangle+a_{2}|2\rangle+\cdots+a_{n}|n\rangle
$$

Crucially, this is a digital representation as each cobit, due to the macrocolumn architecture, resides in separate degrees of freedom. The set $\left\{a_{1}, a_{2}, \ldots, a_{n}\right\}$ of complex numbers are the membrane potentials of each of the $n$ minicolumns which specify the state vector $\left(a_{1}, a_{2}, \ldots, a_{n}\right)$. Together these represent the system in a superposition of the states for which the total amplitude is not in any one definite state (Gudder, 1988). Taken as a coherent unit, these complex amplitudes form the logical unit (the cortical bit). Again, this is a probabilistic model and the amplitudes represent the probability of each state through the normalization rule. This is imposed by the natural oscillations of the inhibitory interneurons due to the relative membrane potentials of the neurons in the minicolumns. The probability amplitudes are then registered as complex numbers in the axonal or dendritic synaptic weights of other cells in the cortical circuit. Membrane potentials (amplitudes) being in a superposition of different states is computationally distinguishable from being in one or the other states. A crucial point to notice here is that the complex numbers can encode states over a continuum ${ }^{18}$.

\footnotetext{
${ }^{18}$ Certainly, down to some physical constant as would seem to be true for any physical system. For all practical purposes ... the setting of a synaptic amplitude input on a dendritic operator may be thought of as programmable over a continuum of possibilities. The means for setting the scalar are changes to the synaptic weight, changes to the dendritic morphology, changes to the position of the synapse relative to others, etc. Thus, unlike a classical binary model of computation that has a finite set of scalars available, this model of computation has "practical" access to a continuum of scalars: the complex numbers. This allows for continuous variables in the model. Continuous-variable information, in this model, can be encoded using the wave-function representation or the phase space formulation (Killoran et al., 2019). In the former, specify a single continuous variable, say $\mathrm{x}$, and represent the state through a complex-valued function of this variable (called the wave function), which is simply a vector in the space of square-integrable functions L2(C). Concretely, $x$ may be interpreted as a "position" for the vector in state space, and (x) 2 as the probability of being "at x". While this is called a "position" in the vector's state space note that it may be for a variable that has nothing to do with "physical space." Standard cortical operators on the state vector can apply (Koch, 1999). However, while this (finite realization of the) model has access to continuous parameters (the complex amplitudes encoded in membrane potentials) those parameters are not directly "observable", effectively shielding (the observer encoded in the model) from the bizarro consequences of the Axiom of Choice and the Continuum Hypothesis (Aaronson, 2006a). The model has the freedom to rotate the measurement basis and represent continuous variables by "accessing" the continuum but it does not "realize" the continuum. Only discrete states are "realized". Having this kind of "access" to the continuum for a "free choice" of measurement basis may be the thing called "freedom" by Conway and Kochen (2009). However, this author would come down on the conservative interpretation that finding this kind of "freedom" in an external "system" would be an artifact of the model of computation itself and that an observer realized within the model of computation would be projecting the model in proposing that "systems" external to the observer also "possess" this inherent character of the model. It may indeed be the case that external systems can possess this model of computation, and perhaps it is merely the best "pragmatic" way of moving forward, but it seems that such a proposition creates a paradox and that a model of computation only ever "sees" systems as having the fundamental nature of its own model of information. The model has by nature a paradox-inducing freedom stated in many places and in many ways - for example, as the "rule-following paradox" (Wittgenstein, 1953). Once an observer creates a measurement
} 
For example, consider the state vector represented in

$$
|\Psi\rangle=\frac{|1\rangle+|2\rangle}{\sqrt{2}}
$$

as a superposition in a 2-cobit. Here each minicolumn state has amplitude $\frac{1}{\sqrt{2}}$ and probability $\left|\frac{1}{\sqrt{2}}\right|^{2}=\frac{1}{2}$ or 50\%. In other words, the minicolumns have equal normalized amplitudes or membrane potentials. With equal potentials they equally inhibit each other placing their firing equally at phase shifts of $\frac{\pi}{4}$ from the peak (or minimum) of the measurement basis. Notice also that the state vector

$$
|\Psi\rangle=\frac{|1\rangle-|2\rangle}{\sqrt{2}}
$$

is the same in magnitude of the amplitudes but differs by a relative phase. In both cases each outcome has an equally likely probability of $\frac{1}{2}$. The relative phase difference is basis dependent. It is always possible to construct an orthonormal basis set of vectors to represent this space (Strang, 1980) and to transform the basis such that the state is expressed only in positive amplitudes (Halmos, 1958). The phase difference encodes the system is being "looked at" differently but still reflects a situation of two equally likely outcomes. Carandini and Heeger (1994) emphasized that to be a true superposition it must depend on the entire population of cells, summed over all possible aspects. Mel et al., (1998) describe implementation of superposition in cells of the visual cortex. These phase-coupled states oscillate together as a coherent unit (Klausberger and Somogyi, 2008; Keane and Gong, 2015).

If the cortex includes in its programmed circuitry a control executive (could be called a user or observer $^{19}$ ) then it must, of course, itself be a part of the overall program encoded in the cortex. Thus, it must reside by this model across particular bases itself. Note that with this type of superposition the observer is not confused about a state. It would be incorrect to say that the state is one, the other, both or neither (Albert, 1992). Stated as simply as possible there are complex-valued amplitudes in mutually inhibitory column's membranes. The observer is not in phase with any of the state's minicolumns when there is superposition.

The above 1-cobit and 2-cobit examples are only the beginning. What is the upper limit? Extending the state space is necessary for describing an unknown state (Kraus, 1983; Royer, 1996). Some neural operations may require multiple populations of cells with jittered thresholds (Suarez and Koch, 1989). Perhaps most significantly for the cortical architecture, ensemble average computing can result in the signal decreasing exponentially as the number of cobits increases (Nielsen and Chuang, 2000). These and other restrictions, such as overhead may mean the typical dimension of a cobit is much less than the number of minicolumns supposedly available in the macrocolumn and cortex as a whole. The extent to which the cortex utilizes complex bits in superposition in a set of coherently oscillating columns is thus an open question (Takeda et al., 2018).

Furthermore, while multi-level spaces $>2$ minicolumns do offer some computational advantage they do not produce a theoretical leap in computational complexity (Yao, 1993; Bernstein and Vazirani, 1997; Adleman et al., 1997). Whether natural selection would find the extra resource overhead and diminishing

basis then it may "determine" (even for probabilities) the "prior existence" of measurements not yet made but the observer nevertheless retains freedom in the measurement basis that can inhibit or collapse previously possible ways of seeing (Le Penne, 2019; Abulof, 2019). [Exit Rabbit Hole]

${ }^{19}$ The physics literature speaks of an observer. The neuroscience literature speaks of a (prefrontal) executive. It should be emphasized that this aspect of the cortex is actively part of the computation. Self-reference is a well-known feature/bug of universal computers. This user/observer is part of a physical computer - a program running in real hardware. As such it is constrained and empowered by the laws of computation (Lloyd, 2013a; Lloyd, 2013b) - specifically, subject to the Turing/Gödel limit of not being able to consistently describe the use of itself (Frauchiger and Renner, 2018). 
return worth it in computational efficiency is unknown. That could be the case, however, for domains where any edge in computational efficiency (even without a full jump to another complexity class) may make a survival difference. For examples of multi-cobit states in cognitive theory see Busemeyer and Bruza (2012). A high degree of network and synaptic plasticity does allow for rapid transformations of these spaces (Koch, 1999). There is the question of to what extent neurons may group to form ad hoc minicolumns, or multi-unit arrays, independent of the minicolumn architecture. So long as such units work as mutually inhibitory logical primitives this probabilistic model could hold.

It may be worth pointing out the obvious that the computational unit of the state vector as described here is only a framework associated with some system. Within the model it can only be stated that the representation of the system in the cobit is what the system is said to be - except for a relative change of basis. At least it is how a user of this cortical device may "speak of" the system. Before it has a representation within the cortical device the "system" cannot be said to have any dimension or a state vector. Once the user has a model $n$-cobit for the system developed then questions may be asked about the adequacy of its dimensional space or consistency with input response - which may lead to revision of the model. The meaning of any information in the model is always entirely contextual by the nature of its structure in the model of the observer (Moreira, 2019). Users/observers are more likely to avoid philosophical inconsistencies by remembering that every system is only known in so far as it is a representation in the cortical model. These are states in a computational model associated with an external (or another internal cortical) system (Fuchs, 2010). All that the observer can know are the representations the model makes of input response. And the model will, of course, ultimately be found by the observer to be the unifying model for representation (explanation) of all inputs.

Now, how does the state vector change? As described above the state vector is the complete complex waveform in all the state space's membrane potentials. These complex number amplitudes are the result of the positive or negative post-synaptic potentials encoded in the synaptic weights along neuronal dendrites. The cortical column is part of a primitive computational unit that extends from its dendritic inputs to its axonal outputs and can be used to form a computational model. The synaptic weights are input to a computational device - input responses that show up somehow, someway, from somewhere outside of the receptive field. Of course, that input may come from other cortical units or recurrently from the minicolumns themselves.

The focus then shifts to what the cobit model can do with the input. There is somewhere on the order of $10^{4}$ excitatory and $10^{3}$ inhibitory inputs on a single cortical pyramidal cell's dendritic spines and shafts. How can this set of complex numbers be mapped into a mathematical formalism? First, consider the function of the unit when synaptic weights do not change.

The mathematical operations described above are linear operators between vector spaces and linear operators have formal matrix representations (Halmos, 1993). An operator is a prescription for how the cortex, using the set of mathematical and logical operations, can make a new vector, that would be new membrane potentials in the minicolumns, out of the old membrane potentials. For this computational model should there be any restrictions on the operators? Certainly, to maintain the map to the model requires an operator to preserve the inner product between vectors - don't break the Hilbert space! The operator and its inverse should return the original state. The operators that satisfy these conditions are represented by what are called unitary matrices. One set of coordinates may be transformed into the other by using a unitary matrix that relates the two sets of basis vectors. Unitary matrices are effectively rotations that preserve the sum of squares, 2-norm (Nielsen and Chuang, 2000). They are operators that do not change synaptic weight encodings. This is the linearity condition (Penrose, 2004). Per the kinds of neural functions available it is possible to program the cortex with unitary operators. That is, a unitary matrix is a mathematical formalism for what can be realized physically in dendritic and synaptic morphology as computational interconnections that map a complex-valued vector in the state space to another complex-valued vector in the state space. Therefore, 


\section{Cortical operators include unitary operators.}

When a unitary matrix is encoded by synaptic weights over the minicolumn's dendrites it can perform an operation on the state vector by encountering the "present state" of the vector. Massive recurrent connections feed back the present state of the unit (Douglas et al., 1995; Douglas and Martin, 2007). This is the membrane potential of the postsynaptic neuron. Thus, the unitary matrix of input may operate on the present state to produce the next state's membrane potential. The effect is to rotate the state vector within the space prescribed by the fixed synaptic weights. This is the second postulate.

Postulate 2: The evolution of a system is described by a unitary transformation. That is, the state $|\Psi\rangle$ of the system at time $t_{1}$ is related to the state $\left|\Psi^{\prime}\right\rangle$ of the system at time $t_{2}$ by a unitary operator $\mathrm{U}$ which depends only on times $t_{1}$ and $t_{2}$

$$
\left|\Psi^{\prime}\right\rangle=U|\Psi\rangle
$$

where $U$ is a matrix representation of the linear operator encoded in the dendritic tree morphology and synaptic coupling connection weights. Scalar products, recorded in synaptic weights, are preserved under unitary evolution.

As with the state space and state vector, the postulates do not provide a priori which unitary operator will describe successfully a system's dynamics! Just as for the state space and state vector, the nature of a system's unitary dynamics is what is meant by, or what characterizes, the system. For 2-cobit systems it turns out that any unitary operator at all can be realized (Nielsen and Chuang, 2000).

For an example, consider the $X$ matrix

$$
X \equiv\left[\begin{array}{llll}
0 & 1 ; & 1 & 0
\end{array}\right]
$$

as the matrix representation of the linear operator that switches or flips the amplitudes on the two 2-cobit minicolumns. In matrix notation, for

$$
\begin{gathered}
|1\rangle=[1 ; 0] \quad|2\rangle=[0 ; 1] \\
X|1\rangle=[01 ; 10][1 ; 0]=[0 ; 1]=|2\rangle
\end{gathered}
$$

This is the matrix formalism for the actual physical process in cortical minicolumns of a 2-cobit interchanging its state amplitudes. The cortex could do this by reversing the input response assignments, put the input through inverter logic, or manipulating the external system - indeed this is what the operator formalism represents taking place. Per the operations above the necessary dynamic neural "VLSI" rules, so to speak, are available to assemble the operator hardware ${ }^{20}$. The matrix formalism is a higher-level way to represent hardware manipulation. Notice this $X$ gate amounts to a 2-cobit NOT gate.

Given

$$
|\Psi\rangle=a_{1}|1\rangle+a_{2}|2\rangle
$$

\footnotetext{
${ }^{20}$ Whatever layout rules exist for the nervous system must ultimately be made possible with the DNA code. This paper is describing the computational model of a device "as found". This would be like describing the model of computation found on a modern microchip while ignoring whatever design or manufacturing processes may have been involved to produce it.
} 
and unitary operator X, the NOT gate,

$$
X|\Psi\rangle=a_{2}|1\rangle+a_{1}|2\rangle
$$

or, in the computational basis

$$
X \equiv[01 ; 10] \quad, \quad X\left[a_{1} ; a_{2}\right]=\left[a_{2} ; a_{1}\right]
$$

The NOT gate can be applied through controlled inhibition on two dendritic branches for each basis vector

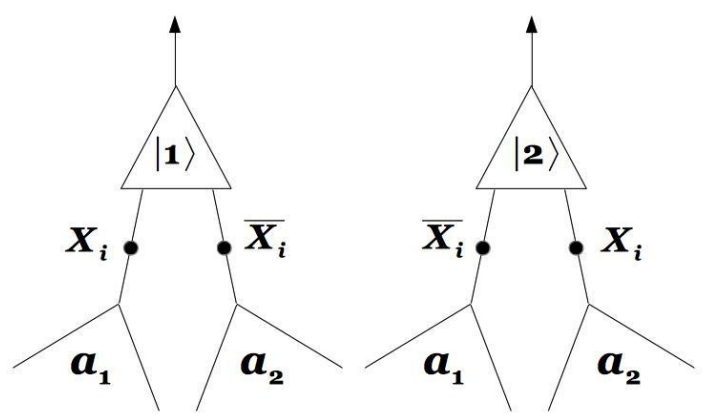

Figure 2. Cartoon realization of the NOT gate in the dendritic arbors of the minicolumn's pyramidal neurons.

Here the $\Delta$ represents the soma of the pyramidal cells. The $a_{1}$ and $a_{2}$ in the branches can be any function or logic (see Koch, 1999). Thus, if operator X is applied, then left and right branches, respectively are inhibited and the result is $a_{2}|1\rangle+a_{1}|2\rangle$. The cortical model is, unlike a classical binary computer, not on a single clock phase. Inhibition of the waveform can occur across phases - a complex number encoding under the 2-norm.

Furthermore, the inverter $\mathrm{X}$ logic can control-U gates such as the conjunctive control-U or Toffoli gate.

Consider Toffoli control minicolumns as shown in Figure 3.

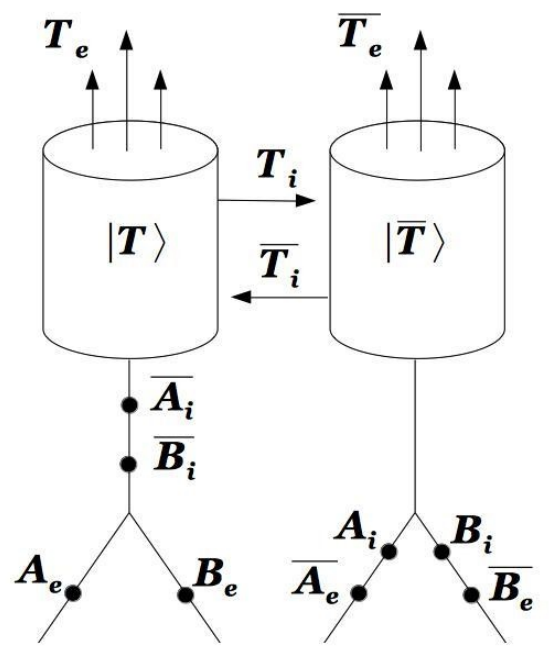

Figure 3. Programming for a Toffoli control gate by placement of excitatory and inhibitory synaptic amplitudes. 
Thus, if (A and $\mathrm{B}$ ) the $\mathrm{T}$ will switch the required amplitudes $|A B X\rangle,|110\rangle \rightarrow|111\rangle$ (see Busemeyer and Bruza, 2012).

Importantly, note that the inhibitory effect is a controllable variable. Meaning, by clustering or separation (among other mechanisms) the effect of inhibition, and thus of the amplitudes, may be variable and not simply all or none. Thus, amplitudes may continue to be superpositions of states. The cortex does not compute on a single phase clock but over a superposition of many phases, themselves embedded in multiple frequencies. Algorithms can thus produce structures on oscillatory interference. Furthermore, remember that these rotations in state space are possible with no changes to synaptic weights. Based on input the device can represent a superposition of possible states or toggle between them (Conte et al., 2009).

These linear operators operate on every component of the state simultaneously by axonal branching and through simultaneous excitatory and inhibitory connections to the dendritic trees of the minicolumns representing the state. The cortical model can thus combine parallelism with complex-number interference along any phase of the membrane potential oscillation. This parallel interference possibly allows this model to compute some things about a state by answering fewer questions than a classical model would need to as in evaluating a disjunction (Bub, 2016). An amplitude can be a linear combination of basis functions, each oscillating at a particular frequency, for the Fourier transform (Moore and Mertens, 2011), an operation tailor-made for efficient computation in the cortex (Koch, 1999). Other algorithms could use this property to efficiently detect periodicity or symmetry.

Programming consists of specifying the operator matrix for each minicolumn to position the state vector within the Hilbert space defined by the minicolumns. The superposition of a state represented in mutually inhibitory amplitudes requires using the vector formalism described in the postulates above. The voltage diffusion within a dendrite is a classically described physical process. However, the mutual inhibition of normalized membrane potential amplitudes across multiple coherent columns maps to a complex-valued "wave" model. This is a very important distinction. The requirements of the model are in the structure of the information primitives - normalized amplitudes used to calculate probabilities in a device capable of unitary transformations. This thesis stands on the postulates of the model (Nielsen and Chuang, 2000).

The primitive cortical bit of information is a complex number encoding. Mutually inhibitory column vectors form under the 2-norm. Unitary linear transformations can be realized in the dendritic morphology for state evolution. This combination of parallelism with interference of complex amplitudes, combining in or out of phase, describes a model of computation. Only these three things are necessary for this model: complex amplitudes, the 2-norm and unitary operators (Von Neumann, 1932; Bub, 2008; Pitowsky, 2008a; Chiribella, 2011; Aaronson, 2013; Moore and Mertens, 2011). There is clear evidence for all three in the cortex.

What about a case where an operator fixes the continuous evolution of the vector in the state space? In the case where the operator reduced the space to a 1-cobit this results in no superposition of interfering amplitudes across outcomes but only a "classical" complex variable for one outcome. One physical mechanism for this would be the disconnection or pruning of the synaptic connections that previously tied together mutually inhibitory columns. As described above, this would be indistinguishable from an architecture with no mutually inhibitory interference. Information is not present in other outcomes, but only as a complex number magnitude in one minicolumn space. Call such an operator, which for all practical purposes removes other outcome spaces in superposition, that removes interference of amplitudes, a "measurement",

\footnotetext{
${ }^{21}$ In honor of Born the rule is named here. Derivable from what Gleason calls frame functions, or the lattice of events in complex Hilbert space, the spherical harmonics for rotational invariance (Gleason, 1957; Shrapnel et al., 2017).
} 
Postulate 3: Any operator on an $n$-cobit

$$
|\Psi\rangle=a_{1}|1\rangle+a_{2}|2\rangle+\cdots+a_{n}|n\rangle
$$

that increases $n$ is called an "expansion" of the state space while an operator that reduces $\mathrm{n}$ is called a "reduction". The case of reducing the state space is also called a "measurement". For the collection of measurement operators $\left\{M_{n}\right\}$ if the state vector before measurement is $\Psi$ then the probability that result $n$ occurs is

$$
p(n)=\left\langle\Psi\left|M_{n}^{\dagger} M_{n}\right| \Psi\right\rangle=\left|a_{n}\right|^{2}
$$

the membrane potential of the minicolumn. The state after a synaptic change is then renormalized

$$
\frac{M_{n}|\Psi\rangle}{\sqrt{\left\langle\Psi\left|M_{n}^{\dagger} M_{n}\right| \Psi\right\rangle}}
$$

The squared modulus rule follows from normalization of total amplitude in the minicolumns. The notation $\langle Y\rangle$ is the inner product on a linear operator notation (Nielsen and Chuang, 2000). In his description of this model, Von Neumann (1955) did not specify the mechanism for measurement. To be clear then, measurement is a physical change in cortical computational hardware.

Measurements discontinuously "jump" the state vector to a new state defined within the projected state space by the new synaptic weight. In the language of linear algebra, a measurement changes the original state vector into a new state vector by projecting the original state into the subspace representing the observed event and dividing the projection by the length of the projection (Busemeyer and Bruza, 2012). Measurable properties of systems are called observables and are represented by linear operators on the vector space associated with those systems (Albert, 1992). A synaptic change represents the arrival of fundamentally new information. It represents the cortex (and possibly user) learning something about the world (or itself).

Measurement operators are a different class of physical mechanism in the cortical hardware compared to the evolution operators. While the evolution operator does not change the synaptic weights over the dendritic trees but preserves the "shape" of the wavefunction, the measurement operator is a discontinuous physical change in the synaptic encoding. The event represented in the measurement operator leads to a membrane potential (firing rate) in only one minicolumn (or subset of minicolumns) for the state of that part of the system. The other previously coherent minicolumns (dimensions) are now silenced and no longer interfere through the normalization condition. There is no longer a superposition from mutual inhibition. And a measurement operator may only be said to have been applied upon such a "collapse" of the state space. This ability of the cortex to dynamically project the state space by expansion or reduction distinguishes it as a computational device, as Koch says, "like a machine that changes its instruction set as a function of its input" (Koch, 1999). Measurement does not reveal some pre-existing value. It is an intrinsically random change to the state vector, it cannot be pre-computed, and it depends on the measurement operator that is "chosen" or that occurs.

The $X$ operator above (one of the so-called Pauli operators) is not only unitary, $X^{-1}=X^{*}$, but also self-adjoint, $X=X^{*}$, so that it also gives an example of a measurement operator that represents an observable. That gives an example of what Dirac (1958) called the eigenvalue-eigenstate link. This says that a system has a property if and only if its state is represented by an eigenvector of the operator. The 
eigenvectors form a basis for the operator. The mini columns are exactly such basis vector representations - the eigenstates of the property. This operator-eigenvector relation does not depend on the basis chosen. The same relation will be obtained on any basis whatever (Albert, 1992).

A related but distinct computation is decoherence. Decoherence is best thought of as phase damping or "phase kicking" (Zurek, 1991; Nielsen and Chuang, 2000). When one minicolumn (basis dimension) controls all the mutually inhibitory interneurons then it will fire at the minimum of the inhibitory oscillation. All other coherently connected minicolumn amplitudes are "kicked down" to coincide with the maximum of the inhibitory cycle. Decoherence then has occurred by the physical mechanism of inhibition or shunting inhibition. The other minicolumn spaces have been squeezed or filtered out leaving a single minicolumn space as the sole "measure". But the n-cobit superposition is still "wired up" with the synaptic weights in place. They are only completely inhibited "as if" pruned. In the matrix formulation the off-diagonal amplitudes are suppressed through inhibition. The "decohered" minicolumn dimensions are silenced by inhibition, except perhaps for background firing (Koch, 1999). And, although a distinct computation, decoherence can have the appearance of collapse, the disconnection of minicolumns. Thus, distinct physical mechanisms among the cortical primitives explain why collapse implies decoherence but decoherence does not imply collapse (Pessoa, 1998; Conte, 2010).

Following a measurement the state vector wave function resumes evolving in its state space based on the linear, deterministic function encoded by the synaptic weights and dendritic morphology. Although measurement (decoherence) is the third postulate the first two postulates depend on this one. Measurement "shapes" the state space for state vectors and operators. ${ }^{22}$ Von Neumann (1955) referred to these two fundamental, yet incompatible, procedures as Process 1 (linear evolution) and Process 2 (nonlinear measurement or "collapse"). The wave function encoded by discrete amplitudes of multiple columns of neurons is not like a classical wave. A good appreciation of the difference can be had by comparing the classical voltage diffusion described by the Pearlmutter and Zador (1999) procedure above to the picture of a vector rotating around in the complex plane. The "waviness" is in the argument of this wave function as its phase. This produces a twisting round and round, corkscrew or wave packet picture (Penrose, 2004). The interference within a dendritic cable is classical wave diffusion but the interference between mutually inhibitory columns encoding a state vector, joined together under normalization, is not "classical" in the physics or computational sense. The entire set of columns can be considered as a single state vector, the logical primitive of the model (Figure 1). It is holistic in the sense that change in amplitude anywhere results in mutual inhibition amplitude everywhere which is not true of classical waves. In the cortical wave function constructive interference and destructive interference are apparently instantaneous to the user. Until a measurement changes the state space the state vector deterministically rotates or fluctuates (evolves) within the probability distribution encoded by the state space of dendritic morphology and synaptic weights.

The commutator of two matrix operators $A$ and $B$ is denoted $[A, B]=A B-B A$. If $[A, B]=0$ then $A$ and $B$ share at least one set of eigenvectors which form a basis. Operator matrices of incompatible observables can't share any complete basis. Thus, the commutators of incompatible observable matrices are nonzero. If knowing the value of $A$ gives the user no information whatsoever about the outcome of measurement $B$ then $A$ and $B$ are maximally incompatible (Bub, 2016). If the user being in an eigenstate of $A$ simultaneously puts operator $B$ in a totally undecidable position (an equal superposition as shown above) then the user and $B$ are maximally incompatible or phase mismatched. ${ }^{23}$

\footnotetext{
${ }^{22}$ This relationship between measurements and the state space is known as the "no-restriction hypothesis." This is that the set of possible measurements is the dual of the set of states (Sainz et al., 2018). Taking an operational approach, the structure of measurements, the Born rule and the post-measurement state-update rule, may be deduced from other postulates, often referred to as "unitary" mechanics and the assumption that ensembles on finite-dimensional Hilbert spaces are characterized by finitely many parameters (Masanes et al., 2019).

${ }^{23}$ A growing body of empirical evidence suggests this model does work in explaining how incompatible representations influence coherence in cognitive heuristics, biases, contextuality, and sequence effects in choice architecture (Jaeger and Trueblood, 2019).
} 
This model allows for correlations to appear that are structurally different from a classical model (Bub, 2016). Observables in different bases will, in general, not commute, meaning $[A, B] \neq 0$. If observables do commute, $[A, B]=0$, then their properties form a Boolean simplex lattice (Pitowsky, $2008 \mathrm{~b})$. But, if the observables do not commute then it is not possible to join them together in one Boolean context. A Boolean logic can't get a grip on non-commuting bases (Kochen and Specker, 1967). Gleason (1957) described noncommuting observables as "intertwined". So, what the cortex can have is a collection of distinct Boolean algebras (bases or perspectives) representing contexts intertwined in a particular way. ${ }^{24}$ Noncommuting minicolumns cannot synchronously (in phase) oscillate together to represent a coherent state when one is being measured, meaning the user is using that basis. The model only relates correlations.

The user could, of course, switch contexts to another basis. But then there is no information about the first. It would not be computationally possible to measure them simultaneously. However, critically important, amplitudes in both bases continue to exist in the membrane potentials and possibly produce interference regardless of the user's basis. If the environment for the cortex consists of correlations that will not fall within a single Boolean context then it stands to reason that this "information gathering and using" device (Gell-Mann and Hartle, 1990) would adapt to allow for alternative perspectives. This would prevent a user from using any particular Boolean logic (like causality) to explain extra-Boolean correlations. The cortex can operate classical Boolean algebras formed over observed amplitudes. However, the cortex can encode a family of intertwined Boolean algebras over observed amplitudes that precludes the possibility of embedding the whole into one inclusive Boolean algebra (Bub, 2019). The cortical user will then be faced with the impossibility of being able to assign truth values consistently in all possible Boolean algebras. This limitation is a consequence of the nature of computation that has been long commented upon under the headings of philosophy.

Very important interpretational note: just as for general states and operators, any measurement operators and outcomes for a system are not encoded in the model a priori. The cortex or user must discover, find, or learn these (Abbott and Blum, 1996; Aaronson, 2007). Indeed, experience teaches the cortex by encoding the measurement operators into the arborizations of the state space's dendrites allowing input to point the vector to the basis state. However, it is certainly possible, as Gell-Mann and Hartle (1990) suggested, that a natural selection process could genetically encode cortical areas downstream from sensory or peripheral input domains preconfigured to adaptive bases. From the user's perspective there are two kinds of processes: (1) unitary evolution and (2) discontinuous jumps to a new state, whereupon evolution begins again. These depend upon the unique contextual, contingent nature of the interaction of the cortex with peripheral input, or neural input, and the nature of the biophysics including the inherently probabilistic operation of the synaptic mechanisms and the condition of health. A measurement operator depends upon the particular basis representation of the system. The cortical encoding of a system is not, of course, the "thing" itself but, as stated in Postulate 1, it is associated with a system by the cortex. By such association to a state space the cortex defines that of which the user may speak (Wittgenstein, 1921) ${ }^{25}$. Furthermore, it seems reasonable that natural selection would adapt a computational device to approach the computational model of its environment if its purpose is to predict that environment (Wigner, 1960).

The intrinsic randomness of the measurement (decoherence) process, which includes the cortex actively "choosing" a basis, is demanded by the nature of computation itself. ${ }^{26}$ A universal computer is a

\footnotetext{
Conjugate coding (Wiesner, 1983) of position and velocity are mapped onto firing rates and co-firing rates of neural spike trains resulting in an uncertainty principle for neural coding (Grgurich and Blair, 2019).

${ }^{24}$ There can be either only one or uncountably many contexts in a spectral effect algebra (Jencova and Plavala, 2018). A convex spectral effect algebra is either classical (the classical probability theory) or has infinite contexts (the non-classical probability theory described in this paper.) The spectral theorem gives that any normal operator on a Hilbert space can be diagonalized, that is, it is unitarily equivalent to a multiplication operator (Bisio, 2019).

${ }^{25}$ Every last scrap of our external experience is of virtual reality (Deutsch, 2011).

${ }^{26}$ For discussion see Svozil (2018) and Landsman (2019).
} 
programmable device that can carry out any algorithm it is given (Moore and Mertens, 2011). Universal models of computation possess a "featurebug" of being self-referential. The cortex faces the problem of choosing a good basis for some measurement relative to the basis or bases with which it represents the "user", a self-referential program running on its own hardware. Decoherence (measurement) should result in a preferred basis for the user, making certain facts determinate from that perspective (Barrett, 1999). But here is a self-referential "strange loop" because a program is calling itself. In Chapter 8 of Albert (1992) it is shown how this self-referencing nature of the model leads to an undecidable, paradoxical situation. This undecidability of a preferred basis is a "feature" because it allows for cognitive adaptation the art of finding a good basis. But that comes with the price, the "bug", two logically contradictory processes, evolution and collapse, that cannot be reconciled. It had to be that way given that universal computing entails such paradoxes! The user may describe the situation as "randomness" or "free choice" in the basis (Conway and Kochen, 2006; Conway and Kochen, 2009; Bub, 2016). The computational consequence is the cortex (user) or natural selection is left with just trying solutions. If the user (cortex) learns something from its perspective it "collapses" to the distribution conditioned on that knowledge, just like for classical distributions (Moore and Mertens, 2011; Busemeyer and Bruza, 2012).

Everett (1973) appealed to Von Neumann's principle of psycho-physical parallelism to "consider the problem of observation itself." He suggested modeling observers as "mechanical devices ... equipped with memory registers ... These (memory) configurations can be thought of as punches in a paper tape, impressions on a magnetic reel, configurations of a relay switching circuit, or even configurations of brain cells ... It will appear to the observer ... that observation caused it to 'jump' into an eigenstate in a random fashion ... Relative to (the observer) the (observed) system states are the corresponding eigenfunctions ... Thus, with each observation (or interaction), the observer state 'branches' into a number of different states ... the 'trajectory' of the memory configuration is ... a branching tree ... In any memory device the branching does not continue indefinitely but must stop at a point limited by the capacity of the memory" (Everett, 1957a, 1957b). It is perhaps unclear exactly how Everett thought of the "branches" (Barrett, 1999). The most conservative interpretation would be to stay within his computing model and think of these as branches in computational trees. He added that "all branches are actual" and the "total lack of effect of one branch on another (in measurement) implies no observer will be aware of any 'splitting' process" (Everett, 1957b). This suggests some sort of inhibition of branches in the model for the measurement (or decoherence) process. The amplitudes interfere with themselves, along the lines of a random walk propagating down a tree (Moore and Mertens, 2011), to mix for search or sampling tasks. Everett never used the phrase "many worlds", although he let it pass without comment in DeWitt and Graham's anthology (Barrett, 1999). With Everett's computational model metaphor, the best explanation for the paradox of Von Neumann's two processes may reside in the nature of computation itself: measurement entails the self-reference of universal computation. ${ }^{27}$

\footnotetext{
${ }^{27}$ This is purportedly something of a "measurement problem." The "problem" stems from the fact that of the many classical probability distributions that are implicit in the state description of this model, the one that emerges is always conditional upon the "choice" of the many possible measurements performable on the system (Janas et al., 2019). The "choice" of measurement context is a computation of the probabilistic model in the predictive device that creates the experiment. A choice is a random sample from a probability distribution. A random event is simply a sample from a probability distribution. In the cortex these distributions may not be static - as Koch said, a machine changing its instruction set as a function of its input (Koch, 1999; Ebert and Wegner, 2011). This model's kind of randomness (freedom in algebraic basis) captures both senses of a lack of predictability and a measure of (un)certainty (Landsman, 2019). Humans are a proven source of true "random" numbers for some computational states - insofar as any sequence may be considered "random". "Certified" randomness is advertised for this model with no assumptions placed on the device's inner workings (Vazirani and Vidick, 2011). The only requirement is the statistical presence of non-classical correlations - which appear in cognitive studies (Basieva et al., 2019). In other words, human beings can be programmed as an entropy source that may be used to generate certified random numbers (Rapoport and Budescu, 1992; Halprin and Naor, 2009; Alimomeni et al., 2013; Sharifian, 2016; Abellán. et al., 2018). Any claim of such randomness is vulnerable to the Bohmian loophole (just like application of the model to randomness from physical systems is vulnerable) which can only be closed with adoption of an extra "principle" (Yearsley, 2019).
} 
Now, it is known that the cortex probably never activates only one column for a complex cognitive state (Llinás, 1985; Tsunoda et al., 2001; Douglas and Martin, 2004; Douglas and Martin, 2007;

Lundqvist et al., 2010). Rather, cortical states are a coherent temporal binding of state spaces (Lundqvist et al., 2006; Klausberger and Somogyi, 2008; Fries, 2009; Vitiello, 2009; Suppes et al., 2012; Keane and Gong, 2015). Following Nielsen and Chuang (2000), beginning with the superposition principle that minicolumns form a linear combination of the basis vectors for the system state then it is possible to form linear combinations of the minicolumn amplitudes of multi-cobit states. Suppose $\mathbf{V}$ and $\mathbf{W}$ are $m$-cobit and $n$-cobit dimensional spaces. Then form an $m n$-cobit dimensional vector space such that its elements are linear combinations of the amplitudes that describe the individual state vectors. Since this involves straightforward complex number matrix multiplication the combined system will have an inner product and inherit the other familiar notions of a Hilbert space described including unitary dynamics and measurement. This operation is known as a tensor product. This is the fourth postulate.

Postulate 4: The state of a composite system composed of distinct state vectors, each encoded in a set of minicolumns, is the tensor product of the state spaces of the component systems. For $n$ systems the joint state can be formed

$$
\left|\Psi_{1}\right\rangle \otimes\left|\Psi_{2}\right\rangle \otimes \cdots \otimes\left|\Psi_{n}\right\rangle
$$

The joint state vector is encoded in a unique set of coherently oscillating minicolumns.

For superposition and interference effects to remain the amplitudes of the state vector of the joint state must be computed in discrete minicolumns. For example, form the tensor product of two 2-cobits. Each 2 -cobit can be said to have two possible states, $|1\rangle$ and $|2\rangle$. A two 2-cobit joint system would then have four possible states, listed side-by-side as 11, 12, 21, and 22. A pair of 2-cobits could then exist in a superposition of these four states utilizing four minicolumn amplitudes, a 4-cobit

$$
|\Psi\rangle=a_{11}|11\rangle+a_{12}|12\rangle+a_{21}|21\rangle+a_{22}|22\rangle
$$

Probabilities and the normalization condition are preserved as before

$$
\sum_{x}\left|a_{x}\right|^{2}=1
$$

Find probability and outcome states also as before. For example, the first 2-cobit gives an outcome of 1 with probability

$$
p(1 x)=\left|a_{11}\right|^{2}+\left|a_{12}\right|^{2}
$$

leaving the post-measurement state

$$
\left|\Psi^{\prime}\right\rangle=\frac{a_{11}|11\rangle+a_{12}|12\rangle}{\sqrt{\left|a_{11}\right|^{2}+\left|a_{12}\right|^{2}}}
$$

Observe as well that the joint state could undergo a synaptic or pruning operation. The four state joint state vector $|\Psi\rangle$ above could undergo a state reduction operation from a four minicolumn to a two minicolumn superposition such as, for reduced state $\left|\Psi_{R}\right\rangle$ 


$$
\left|\Psi_{R}\right\rangle=\frac{|11\rangle+|22\rangle}{\sqrt{2}}
$$

This type of joint state reduction results in what is called "entanglement". There is no equivalent notion to this for "classical" variables (Jozsa, 1997). In the cortical model it is realized as two mutually inhibitory minicolumns, a 2-cobit. It is something like a more efficient encoding of a state space when there is no amplitude evidence for other terms. A measurement on either of the two minicolumns thus encodes the "entangled" outcomes for the two encoded 2-cobits. And, as before, measurement "destroys" coherence and interference. Inhibitory decoherence or synaptic depotentiation can put it on hold over a wide range of time scales.

Because entanglement is a more efficient encoding it may offer some computational and communication advantages (Nielsen and Chuang, 2000; Nelson and McEvoy, 2007; Kempe et al., 2008; Gisin, 2014). This comes at the cost of being able to say anything about the probabilities of the entangled cobits separately. Recall that the joint state vector is now a superposition of the amplitudes that represent the combined states in mutually inhibitory, interfering minicolumns. Until a measurement occurs the total amplitude can only be said to be "spread out" in the membrane amplitudes of the minicolumns. ${ }^{28}$ These states also oscillate coherently as those described above. The finite physical dimensions of the cortex certainly impose some upper bound on the possible dimensions in a joint tensored state.

Above was given an example of how inhibitory and excitatory synaptic weights applied to a minicolumn's dendrites can change values to realize the $\mathbf{X}$ or NOT operator. This unitary operator can be thought of as a computational "gate", a manipulation that converts information from one form to another. In a similar manner the available set of mathematical and logical operators in the cortex can be used to construct other single 2-cobit gates such as

$$
H \equiv \frac{1}{\sqrt{2}}[1 \quad 1 ; 1-1] \quad, \quad S \equiv[10 ; 0 i] \quad, \quad T \equiv\left[10 ; 0 e^{\frac{i \pi}{4}}\right]
$$

which are known as the Hadamard, phase, and $\frac{\pi}{8}$ gates, respectively (DiVincenzo, 1998). Remember that the cortex has the physical mechanisms available to "wire up" phase change operators.

The proto-typical multi-cobit gate is the controlled-not or CNOT gate, a generalization of XOR. This gate can be constructed from two 2-cobits, known as the control and target. The formal matrix representation for the 2 -cobit case is

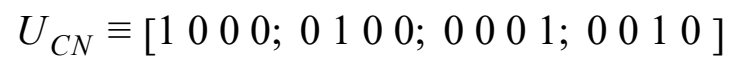

It can be shown that these four gates above form a universal set of gates for 2-cobit computation (Boykin et al., 1999). Recall that the set of measurement operators reduces the state vector to a 1-cobit with no coherent oscillations or interference, that is no complex phase information binding, with other minicolumns for that system state. If constructing a circuit of many gates that includes such an operator it can always be moved to the "end" of the circuit (Nielsen and Chuang, 2000). Otherwise the measurement operator destroys potentially useful coherent information encoded in the phases.

Importantly, a universal gate set allows for the formation of a programmable device that can carry out any algorithm. Unfortunately, although this is a universal set for approximating any unitary operator, constructing such gates is generically a hard problem. In fact, most unitary gates can only be

\footnotetext{
${ }^{28}$ It is argued that the partition of a system into subsystems is dictated by the set of operationally accessible interactions and measurements. The emergence of a multi-partite tensor product structure of the state-space and the associated notion of entanglement are then relative and observable-induced (Zanardi et al., 2003).
} 
implemented very inefficiently (Nielsen and Chuang, 2000). This may be evident in the difficulty in finding, recognizing, or learning an operator that will transform a state.

Has this paper described a universal model of computation? There are assemblies of discrete sets of physical components, the cortical minicolumns, which can describe computational procedures. These operations, such as the $\mathbf{X}$ matrix represents, may be considered as equivalent to gates in a circuit model (DiVincenzo, 1998). A cortical circuit would then operate on some number, $m$, of cortical bits. A cortical bit, the cobit, is a set of mutually inhibitory minicolumns representing all possible states of the system. Where $n$ is the largest dimension of $m$ cobits, the state space is in general an $n^{m}$-dimensional Hilbert space. Product states of the form $\left|x_{1}, \ldots, x_{m}\right\rangle$ are known as computational basis states. The vector $|X\rangle$ denotes a computational basis state, where $X$ is the number whose $n$-nary representation is $x_{1}, \ldots, x_{m}$. Any computational basis state can be prepared in at most $m$ steps by setting the membrane amplitude via the synaptic weights. Such gates can be applied to any subset of $n$-cobits and a universal family of such gates can be implemented (DiVincenzo, 1995; Boykin, 1999).

Information processing is always subject to physical constraints. The number of minicolumns in the cortex is clearly finite. The bounds on the possible space dimensions, product states, and overhead costs are unknown. The cortical component's time constants are very slow compared to CMOS technologies. And the cortical architecture is set up for massively parallel algorithms typically executed in one or two time constants rather than serial processing (Koch 1999). The general $\geq 2$-cobit model is cooperative and dynamic across phases (bases) rather than a single phase like a classical binary computer. Without some cooperative temporal framework the various synaptic potentials would decohere and lose their meaning (Klausberger and Somogyi, 2008).

There are results for the computational complexity of this model (Yao, 1993; Bernstein and Vazirani, 1997). And while some speedup results by increasing the cobit dimension beyond a 2-cobit this does not make a difference from the theoretical point of view for the complexity class of multi-cobit spaces (Nielsen and Chuang, 2000). It is presently unknown if the nature of the cobit makes a difference in complexity classes - meaning it could solve some problems more efficiently than a classical binary computer. There is some evidence for that (Simon, 1997; Grover, 1997; Watrous, 1999). The reduction to the efficiency of entanglement may not be possible for exclusively binary models. However, the potential improvement in efficiency seems to only exist for certain classes of problems - generally, search and period finding. Phase encoding is the lever which enabled progress for many of these algorithms (Nielsen and Chuang, 2000). Efficiency gains for intermediate complexity instances in a class of problems does not necessarily grant efficiency for the so-called "complete" or hardest instances. The question then is if the extension to a more efficient model for problems over the dimensions available to the cortex provides enough of a difference for natural selection to work on. Given the potential importance of search and period finding in natural processes critical to survival Nature may find this adaptive and leverage such a difference even if the speedup were only polynomial (Keller and Klein, 2019; Busemeyer et al., 2020).

It may very well be the case (indeed would seem likely) that the implementation of this model of computation in the cortex does not realize efficiency greater than that of classical computers with physical components that are many orders of magnitude smaller and faster for many problem domains. It is entirely possible to have a specific actual realization of this model of computation that does not realize a supremacy in efficiency to some realizations of the classical model whether or not the two models prove to be equivalent in general. Likewise, it is possible that there are some problems for which this model is more efficient (perhaps search and period finding) than the classical model with a comparable number of devices and with comparable switching speed of computational primitives. 
David DiVincenzo (2000) gave requirements for a physical realization of this model of computing:

1) Scalable physically to increase the number of bits

- This model has dynamic cortical network scaling (Llinás, 1985; Koch, 1999; Douglas and Martin, 2004; Lundqvist et al., 2010).

- Evolution is believed to work on the cortex by adding minicolumns (Mountcastle, 1997; Buxhoeveden and Casanova, 2002a; Hosoya, 2019).

2) Bits can be initialized to arbitrary values

- Synaptic weights and dendritic tree morphology provide a means for setting the membrane potential amplitude (For details of the physical mechanisms, see Koch, 1999.)

3) Gates faster than "decoherence" time

- There exists robust evidence from cognitive science that cortical circuits can maintain unitary coherence of vectors with normalized amplitudes with data confirming non-classical correlations (Tsunoda et al., 2001; Tam, 2002; Ward, 2003; Behera et al., 2005; Bartos et al., 2007; Aerts 2007; Fries et al., 2007; Klausberger and Somogyi, 2008; Aerts, 2009; Conte et al., 2009; DeBarros and Suppes, 2009; Fries, 2009; Khrennikov, 2009; Sun and Dan, 2009; Cheon and Takahashi, 2010; Aerts et al., 2011; Busemeyer and Bruza, 2012; Keane and Gong, 2015; Busemeyer and Wang, 2017; Pothos et al., 2017; Trueblood et al., 2017; Chauhan et al., 2018; Muller et al., 2018; Wojciechowski and Pothos 2018; Broekaert et al, 2019; Cervantes, 2019; Fell, 2019; Qin, 2019). Decoherence increases entropy (Zurek, 2003). But entropy is anthropomorphic (Jaynes, 1965). ${ }^{29}$ As suggested above, it may be best to view "decoherence" as a class of operator (an improper mixture) in this model - making decoherence a functional requirement. It is simply not the case that the decoherence argument prevents realizations of this model of computation outside of certain physical systems or scales (Kastner, 2016). As a computer engineer, this author fully appreciates that realizing models of computation with particular physical systems involves the challenge of engineering fault tolerance in the face of system noise (Vainstein and Jones, 2006). But abstract models of computation are radically independent of any particular physical system or realization (Moore and Mertens, 2011).

4) Universal Gate Set

- Arithmetic, phase, and logical operations available (Koch, 1999) can be used to construct linear operators that form a universal gate set (Boykin et al., 1999).

5) Bits can be read easily

- The set of measurement operators produce a minicolumn population amplitude as firing rate code available to user or other cortical areas through axonal and interneuron projectors (Rieke et al., 1997; Koch, 1999; Molnar et al., 2008).

\footnotetext{
29 "The notion of locality, and therefore the notion of entanglement and what you consider the structure of your spacetime is observer dependent. It depends on your choice of observables. There is a freedom in choosing that. And this freedom can be seen as a freedom in the way you perform partitions. ... The fact is if you want to talk about entanglement entropy you need to say explicitly with respect to which observables the entanglement entropy is defined.... The tensor product structure and entropy are observer and observable dependent. Entropy and the directionality of time, that is always related to the direction you see entropy growing... is something that is observer/observable dependent." (Vidotto, 2020)
} 
It seems reasonable that if a computer engineer were given a microprocessor and asked to identify the model of computation that a conclusion that it implemented a classical binary model of computation could be believed based solely on an argument that the devices and architecture satisfy the classical binary postulates even if they did not apply power and did not observe any actual experimental outcomes. If experimental data collected by the above cited cognitive scientists was given to a physicist and told that it was "the outcome of a physical experiment" the physicist would very happily conclude that the data must be from a non-classical system. In other words, conduct the following experiment: In one lab measure "the outcome of a series of three choices of Stern-Gerlach arrangements on a beam of particles" while in another lab measure "the outcome of a series of three questions on trustworthiness" (Fell, 2019) or the experimental results of Broekaert et al. (2019) or Uprety et al. (2020). If the data from the experiments is then sent in a blind fashion to a physicist in a third location the physicist must conclude that each experiment is measuring non-classical correlations.

There are no "physical" or "scale" requirements in the postulates for this model of computation" (Nielsen and Chuang, 2000). Defining (choosing) an experience (experimental) framework (context) and assigning meaning to its data are computational activities that take place within the model of computation - specifically, for human beings, in the cortical model of computation. The Stern-Gerlach experiment is an example of an experience that reveals the nature of information in the model of computation generating the experience through its organizational architecture. This is true at the most fundamental level about all information for a computational device. The Stern-Gerlach construction projects a model in the same way that the transistor projects a classical binary model. The Stern-Gerlach apparatus was no more a "discovery" of a non-classical model of computation in a "physical system" than the transistor was a "discovery" of a classical model of computation in a "physical system." It may be more appropriate to say it was a discovery of how to project a model onto the organizational architecture of the experience. In neither case is it sensible to say that either model of computation is what the device or "physical system" is "doing" or even "realizing". At the most what may be said is that these are experiences onto which are mapped models of computation - in these examples the latter classical is a subclass of the former

30 “ ... if quantum mechanics isn't physics in the usual sense -- if it's not about matter, or energy, or waves, or particles -- then what $i s$ it about? From my perspective, it's about information and probabilities and observables, and how they relate to each other. Quantum mechanics is what you would inevitably come up with if you started from probability theory, and then said, let's try to generalize it so that the numbers we used to call "probabilities" can be negative numbers. As such, the theory could have been invented by mathematicians in the $19^{\text {th }}$ century without any input from experiment. It wasn't, but it could have been." (Aaronson, 2006b). In the survey by Schlosshauer et al. (2013) only 15\% of the quantum foundations researchers felt that the concept of "decoherence" solves the inherent paradoxes of the model. Meanwhile the decoherence story is accepted as correct and mistakenly thought to rule out realizations in cortical hardware (Tegmark, 1999; Koch and Hepp, 2006). It is often assumed that this model of computation is some kind of superpower that would allow the brain to access supernatural computing powers and, thus, it is preposterous to even consider such a silly thing. However, this model of computation is simply an extension of probabilistic computing to computing with complex amplitudes. It may give some advantages for some types of problems but even those are only some specialized possibilities. Scott Aaronson said it best, "If only I could make one or two people's eyes light up with comprehension about the fallacy of a QC simply trying all possible answers in parallel and then magically picking the best one, or about the central role of amplitudes and interference, or about the "merely" quadratic nature of the Grover speedup, or about the specialized nature of the most dramatic known applications for QCs, or about the gap between where the experimentalists are now and what's needed for error correction and hence true scalability, or about the fact that "quantum supremacy" is obviously not a sufficient condition for a QC to be useful, but it's equally obviously a necessary condition, or about the fact that doing something "practical" with a QC is of very little interest unless the task in question is actually harder for classical computers, which is a question of great subtlety ..." (Aaronson, 2020). It is easy to assume that because the model is realizable (and was first recognized) in "atomic system" devices that this must restrict it to only these devices. The "discoveries" of the model "in" atomic systems may be reframed as the "realization" of the model of computing "in" the experimental constructions that realized the model. That is, the double-slit experiment (and others) are ways to realize computational devices for this model of computing - that is one way to describe what the double slit experiment is... It would not be surprising if constructing computational devices for this model of computation out of human-engineered atomic scale devices turned out to be more powerful than the model using neurons, synapses and dendrites. But, then constructing the classical model out of human-engineered atomic scale devices (doping semiconductors) also may lead to a somewhat more powerful computing device - for some tasks... It is possible that evolution settled on this model for its ability to solve period-finding problems in natural systems in the remote past while the ways it is used now by the cerebral cortex could be less than the most efficient application. 
non-classical. The logic is how the observer manipulates information within a model of computation. Observation is an operation within the model of computation of observers which are themselves computations within the model of computation. The model of computation computes both the experience and the observer. It is not clear to this author if it is ever possible for an observer generated by a particular model of computation to experience information in a way other than as it is framed by that particular generating model of computation. If that is the case, then it would always appear to any observer that their particular model of computation must be the underlying (world) model of computation in which the observer is embedded. It would appear to the observer that all experiences (experiments) return information that is best explained with the logic of the model of computation that is itself generating the experiences of the observer. Of course, the "device" that is computing the observer and the experiences of the observer may be embedded in some model of computation itself that is a superclass of the observer's model of computation. (Within that superclass lower classes may evolve.) But, how could the observer experience (and thus represent) information that does not lie within the boundaries of the observer's model of computation which generates all of the observer's experience? If observers require that their reality (the totality of experience) be explained by a model of computation then the observers will always find their own generating model of computation to be the "final" theory. This is not without great practical usefulness to observers, especially for modeling regions of the superclass that are within their own observer class, but it is always strictly an explanation of their experiences, not of anything beyond their experiences. ${ }^{31}$ The discovery of such a theory then would be discovery of what observers' experiences are in the most fundamental way that the observers may know.

There are mathematically equivalent alternative statements of this model of computation and of the four postulates given above (Nielsen and Chuang, 2000). For example, the density operator (matrix) picture could be an especially helpful device for description of subsystems and approximate methods when a full specification is not available. Major computational benefits could be tapped with a rough quantification of rule strength (Pearl, 1988; Gudder, 2008). In the neural architecture individual Hebbian synaptic weight changes would be the partial trace (Nielsen and Chuang, 2000) of observable quantities for subsystems of a composite system. There is a superoperator formalism that generalizes the second and third postulates. The starting point can be a generalization of the classical Boolean lattice (Niestegge, 2020). But this is left open here if or when one of those may provide a better picture of cortical states and operations.

This paper has described the cortical model of computation. Mutually inhibitory, coherently oscillating columns of cells realize the logical primitive. The variable present in the columns is a vector of complex number valued amplitudes. This complex number encoding that includes negative amplitudes results in interference that is fundamentally non-classical. Logical and mathematical operators are programmed in synaptic weights over various morphological arborizations. These operators, which can be represented as matrices of complex numbers, form a universal gate set completing the model of universal computation in the cortex.

\section{Part 4: Classical Computing with Levers, Marbles, Crabs and 1.4 nm Die Shrink}

The model of computation is often confused with particular physical realizations. For example, the historical contingencies for the model of computation described in this paper, the random application of it

\footnotetext{
31 "Particularly in physics, this development has reached a peak in our own time, in that, to a large extent, the possibility of knowledge of the objectivisable states of affairs is denied, and it is asserted that we must be content to predict results of observations. This is really the end of all theoretical science in the usual sense (although this predicting can be completely sufficient for practical purposes such as making television sets or atom bombs).” (Gödel, 1961). "All models are wrong, but some are useful" (Box, 1979).

${ }^{32}$ Einstein almost certainly meant something else by this reported statement but it applies well here: "Whether you can observe a thing or not depends on the theory which you use. It is the theory which decides what can be observed." (Einstein, 1926/1990).
} 
first in the domain of physics, can lead some to defend certain physical systems as being exclusive for the model. A physical realization of a model of computation is always a projection of the logic of the model onto the physical system. The postulates of the model (mapped in this paper onto non-controversial cortical functions) make absolutely no claim about restriction to any particular physical system, types of systems, or temporal or spatial scales of systems. In the canonical text for this model, Nielsen and Chuang say it best, the model is " ... a mathematical framework for the development of physical theories. On its own (it) doesn't tell you what laws a physical system must obey, but it does provide a mathematical and conceptual framework for the development of such laws." (Nielsen and Chuang, 2000). It is indeed a conceptual framework - literally an arena in which predictive models of experience are developed.

In order to appreciate how radically general is the notion of a model of computation a few examples of physical realizations of the classical model of computation are offered here. For other examples, including computing with the Game of Life and planar tiling see Moore and Mertens (2011).
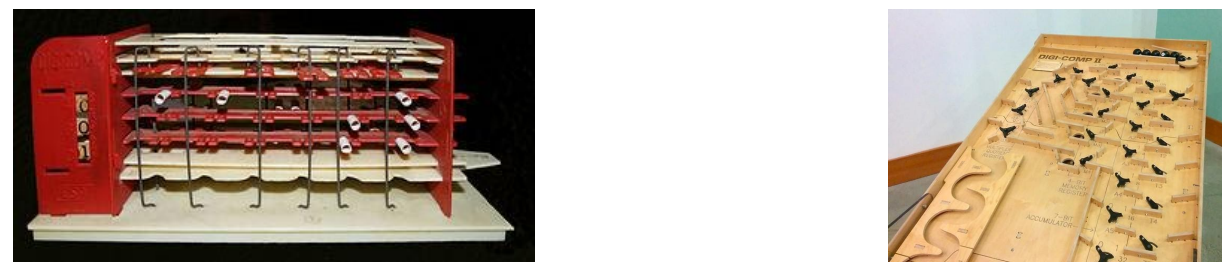

Figure 4. The Digi-Comp I and Digi-Comp II mechanical digital computers. The clock of the Digi-Comp one is created by moving a mechanical lever back and forth by hand. Scott Aaronson described how to adapt the composition of the Digi-Comp II for universality (Aaronson, 2014).
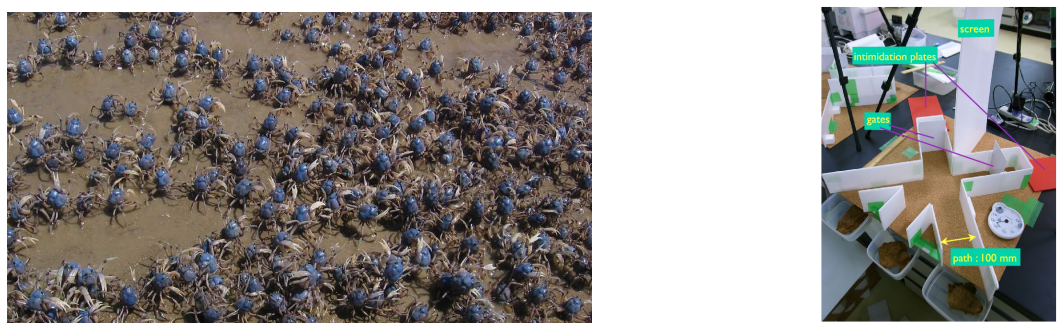

Figure 5. Mictyris guinotae, soldier crabs swarming. Robust soldier crab ball gate. Swarms of soldier crabs can implement logical gates (Gunji et al., 2012). As with the mechanical computers above achieving universality would be a matter of composition of the gates and crabs.

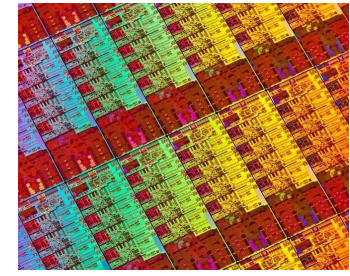

Figure 6. Integrated circuit die. Intel reportedly plans to reach the $1.4 \mathrm{~nm}$ node by 2029.

The point being made here is the model of computation does not determine a physical realization. The classical model of computation is not a marble, a crab, or a transistor. The non-classical model of computation is not, as Aaronson says, "matter, or energy, or waves, or particles." Complaining about the complex-Hilbert, non-classical model of computation of the cerebral cortex not involving "atomic particles" is like complaining that someone referred to their laptop as a classical computer because it doesn't have crabs for logical gates. Likewise, it would be as wrong to presume that to find a classical computer one must find a transistor as it would be wrong to presume that to find a non-classical computer that one must find a photon. 
When a computer engineer is designing a classical computer out of marbles on a Digi-Comp table or out of crabs with crab ball gates or out of transistors on a $1.4 \mathrm{~nm}$ die shrink integrated circuit they are working within the arena of the classical model of computation. When the cerebral cortex constructs minicolumn circuits or inter-column circuits or attractor networks with mutually inhibitory normalized complex amplitudes in membrane potentials and dendritic arborizations it is working within the arena of the non-classical model of computation.

The good way to appreciate just how suspicious one should be about the argument that there is only some limited scale or type of system that can implement a particular model of computation is to imagine there is a planet where the inhabitants have only ever constructed computers out of swarms of soldier crabs. This may require vast areas of land and billions upon billions of soldier crabs passing through crab ball gates. It may not even be very fault tolerant but maybe the inhabitants have achieved at least enough to repeat calculations and achieve fault tolerance by the threshold theorem. For the inhabitants of this planet a "calculator" may be the size of Texas. Now a human arrives with a smartphone and declares that inside that device is the same model of computation that underlies the crab ball computers. The human is promptly fed to the crabs.

The broad notion of universal computation, like Turing's, is extremely fundamental to the point of being trivial (Moore and Mertens, 2011). The only requirements to establish a physical realization of the non-classical model described here are

1. A complex-valued (Hilbert) state space.

2. Normalization.

3. Universal gates.

The neuroscience community sees these facts about cortical computation as being

"known for some time now ..."

\section{Part 5: A Sketch of the Logical Primitive}

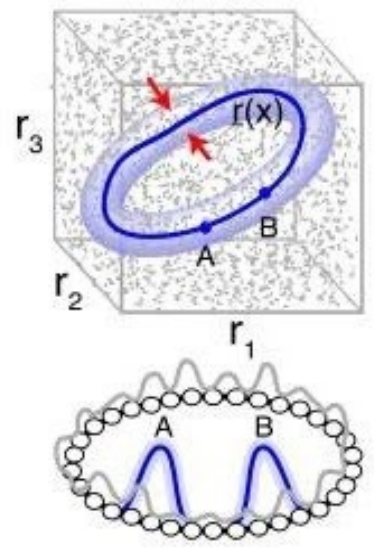

This figure is a good start for picturing what the computational primitive would look like in the cortex. However, it is only a start because the actual encoding of amplitudes in a cortical primitive would more resemble a wave function inside a hologram because of phase information that is not captured by this "frozen" slice shown here. This is part of a figure taken from Yoon, et al. (2013) who describe how a coherent coupling of neuronal amplitudes form a logical primitive state space: "Top (state space): The state of $N$ independent neurons, each described by a firing rate may lie anywhere in an $N$-dimensional cube of side length (shown for $N=3$ neurons). Appropriate coupling between the neurons can shrink the 
allowed states to a low-dimensional attractor (dark blue). All other states are transient, rapidly decaying back to the attractor, and are thus rarely seen. States very close to the attractor (light blue), though transient, may be observed if perturbations frequently drive the system into those states. Bottom: An example network of $N$ neurons (small circles) with 1-d continuous attractor dynamics. Local excitatory and global inhibitory connections (not shown) between all neurons stabilize population states that are local activity bumps (e.g. blue bump A or B; gray: transient/unstable activity profiles). An activity bump is a single point on the continuous attractor (top) of all possible translations of the bump. If points on the attractor are identified with values of some circular variable, then all neural tuning curves for that variable will be identical, except for a phase shift (translation)."

These bumps have been studied primarily as part of circuits encoding spatial location or direction (head cells, for example) which would map directly onto classical spaces. However, these primitive units can easily represent superpositions between possible states for some system such that a non-physical or non-actual superposition would represent a probability between physical or actual system states. For example, using Yoon, et al.'s figure, if A and B above represent orthogonal physical outcomes then a superposition "between" those two possible outcomes would place the neuronal amplitude, the "bump", between A and B, positioning the state vector between measurable basis outcomes.

Here is how to understand the "cube" or "block" space within which the wave function takes shape. The space is some region of the cortex composed of groups (columns or units) of neurons over a receptive field. In the absence of some imposed algebraic basis for measurement the space is filled with completely random neuronal firing. However, once an eigenvector basis is imposed then the synaptic amplitudes can "learn" a Hamiltonian for encoding the wave function of the associated system in the dendritic circuitry. The cortex has a "freedom" to choose an algebra of observables. Without this "observer" choice the Hilbert space is trivial (Vidotto, 2020). Note that there are always non-zero probabilities for firing anywhere in the state space.

The low dimensional attractor is the wave function of the cobit - the "shape" of the oscillations that represent the probability of the cobit being observed in these states. This cortical unit of information allows for representation of amplitudes in single bumps, multiple bumps or as a regular field of bumps with the potential for a rich spectrum of dynamics (Wang et al., 2015; Avitable, 2017). Remember that underlying the normalized probabilities of the system are the positive and negative complex number amplitudes input to the dendritic computations. The deterministic evolution can include random bump walking or oscillating between possible states (Fung et al., 2009; Kilpatrick and Ermentrout, 2012). The underlying model of computation is a probability amplitude wave function (Carroll et al., 2014). Tanaka and Nelson (2018) studied Anderson localization understood to be an interference phenomenon for local excitation and global inhibition ring attractor global neural network models. 
Part 6: What the Computational Model Looks Like in the Representation of Spacetime in the Entorhinal Cortex and for Bosons in an Optical Lattice
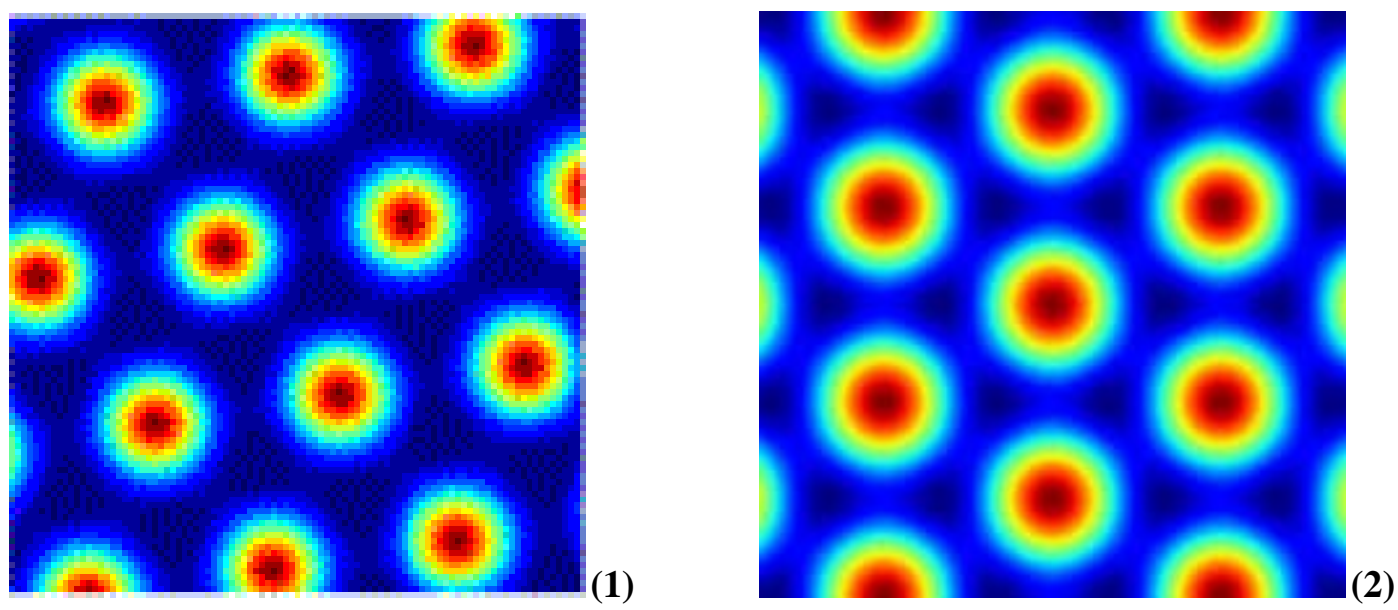

The left image (1) is of entorhinal cortex grid cells creating a computational representation of space (See any of Moser Group, 2019). The right image (2) is of ultracold atoms in an optical lattice (For one example, Anisomovas, 2014). Both are images of "probability amplitudes" formed in a particular kind of computational substrate. In the entorhinal cortex inhibition from propagating neuronal interference aligns grid amplitudes in "bumps". In the optical lattice interference of counter-propagating lasers confines the amplitudes of bosons to potential minima or wells.
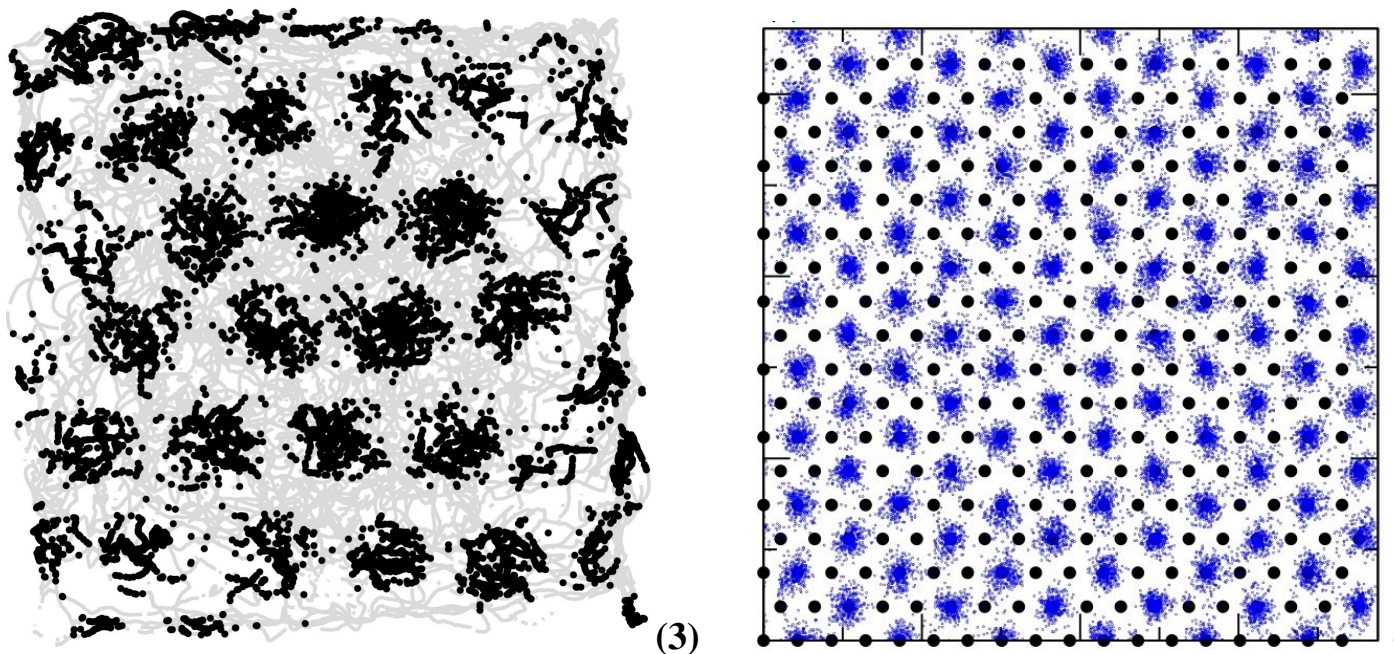

The image (3) on the left is a typical picture record of entorhinal grid cell firing (See any of Moser Group, 2019). The image (4) on the right is an image of particle world lines for a system of dipolar bosons in a two-dimensional optical lattice (Cinti, 2015). In both cases the higher probability of "firing" at that location occurs in the "bumps" or "wells" of the lattice. The lattices can be described by classical models. The interference of underlying amplitudes follow a non-classical model.

Notice that (1) and (2) are models of the amplitudes of the probability wave functions. What actually is seen for any one particular measurement are individual "firings" or "world line projections" shown in (3) and (4). In each realization the wave function is encoded in the organizational architecture of the computational substrate and determines the "firing or location" probabilities. Both result from the same underlying model of computation. The model of computation is substrate independent. 


\section{Part 7: Excerpt of Podcast Interview with the Cognitive Neuroscientists J. von Neumann and N. Bohr.}

Interviewer: Gentlemen, thank you for taking time from your busy schedules to speak with us here today. You both played a role in developing the field of Probabilistic Cognitive Neuroscience. The spooky thing to some people is that this new theory claims we think in a way that involves negative numbers underlying probabilities. Your colleague R. Feynman even said that nobody really understands it.

Bohr: It is not important if someone thinks they understand it or not. It is just the way the model works. And it is not just negative numbers ... the cortex stores information as amplitudes complex numbers. The key is mutual inhibition between neural amplitudes that are stored as membrane potentials. They are like different possibilities that can interfere with each other to cancel out. That is the sense in which they can be negative. The fact is that before there were paradoxes that could not be resolved. But now we can explain cognitive results with this model. I tell my young neuroscience students they must not worry if it seems to not make practical sense. Just do your calculations and do not go down philosophical rabbit holes. This is just the way of doing sober neuroscience.

Von Neumann: The mathematics is the evidence. If the mathematics explains the data observed then that is all we can ask of a model.

Interviewer: There have been some from outside neuroscience who claim that they can use this probabilistic model of computation to explain other phenomena. There are even some physicists who have been called cranks claiming that this model explains the workings of Nature.

Bohr: That is absurd. Anyone who could believe such a thing is either a crackpot or is senile. Such a ridiculous assertion does not even deserve an answer. I mean this is a computational model that works over a continuum of scalars. (Well, down to some constant, anyway.) So, where exactly would the physicist propose to hide a continuum of scalars? In some microscopic particles? It is ridiculous. Do physicists think that there is Hilbert space inside a photon? An electron? An atom? Or someone's pet cat? It is people like that who give science a bad name and mislead the public. Uninformed people think that just because this new theory of probability is mysterious and just because physics is mysterious that they must have something to do with each other. The physicists should stick to Newton and leave computational probability theories to the experts.

Von Neumann: Turing defined what we understand to be computing from observing human beings computing. Now what kind of sense would it make to suggest that a model of computation could have something to do with a non-cognitive science like physics? Would any

\footnotetext{
${ }^{33}$ In one survey of quantum foundations researchers $18 \%$ preferred a many-worlds interpretation (Schlosshauer et al., 2013). One interpretation of the many-worlds interpretation is that all possible universes may/will exist. Thus, we can imagine that a universe exists where cognitive science (and not physics) was the first branch of science to "discover" the "quantum" model of computation. Of course, in that universe the model was not discovered coming out of work on black body radiation or the photoelectric effect and, thus, did not acquire the misleading name of "quantum mechanics." In that universe, cognitive scientists would have been more likely to have named it something like "Interference Cognition" or "Amplitude Cognition" since it is the interference of complex amplitudes that gives the model its character. In that universe the poorly funded field of physics is barely considered a "hard" science and physicists who suggest that the "Interference Model of Cognition" has something to do with the mysterious behavior of light are laughed at for suggesting that light can somehow "think" like people do. The leaders in cognitive science, considered the canonical examples of scientific geniuses, explain that all serious scientists recognize that light may only appear to exhibit such effects because, obviously, it is the human cortex that is processing the experience.
} 
serious person ever think that some state space defined for an elementary "particle" could be a unit of information for a probabilistic computer? The model must realize amplitudes that are used to calculate probabilities. Then the primitive units of information must be inside of an actual computational device that is capable of unitary transformations. Where exactly are these imaginary number devices the physicists are claiming exist?! Are these calculations being done in parallel universes? Or maybe there is a parallel universe where Bohr and I are physicists who ridicule cognitive scientists for trying to use this model of computation to explain neural computations ... (Laughter with Bohr) The devices must produce data outcomes that cannot be modeled with a classical model of computation. You can't just stick the model over some mysterious observation like the physicists do and claim there is "real computing" going on. It does not add explanatory power if you just stick complex numbers and Hilbert spaces onto random physical systems. You must see the computational device that is encoding a complex amplitude! Neuroscience is a venerable field that has been around for hundreds of years. Physicists have only recently begun to image these "particles" they are talking about. Look, physics is important for understanding how to launch cannonballs and giving cute stories about why apples fall. But it is not neuroscience.

Bohr: Imagine we build an artificial intelligence with this model of computation. Let us name it Born. We send it on a thousand-year journey across the cosmos. It returns to us and we ask what it learned. It says:

"To understand the universe, I repeated measurements many times. What I find is that when an observable corresponding to a self-adjoint operator $\hat{A}$ is measured in a state $|\Psi\rangle$, the result is an eigenvalue $A_{i}$ of $\hat{A}$ with probability $p_{i}=\langle\Psi\rangle^{2}$. This must be the model of the universe."

Should we say that our artificial intelligence has been doing physics? 


\section{Part 8: The comic strip version.}

\section{Dpistemologies}
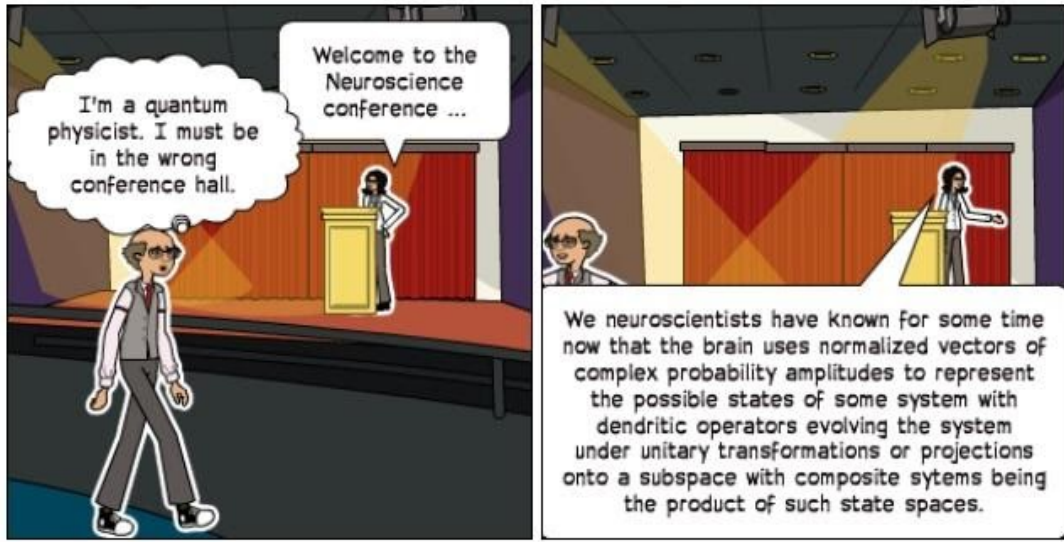

We neuroscientists have known for some time now that the brain uses normalized vectors of complex probability amplitudes to represent the possible states of some system with dendritic operators evolving the system under unitary transformations or projections onto a subspace with composite sytems being the product of such state spaces.
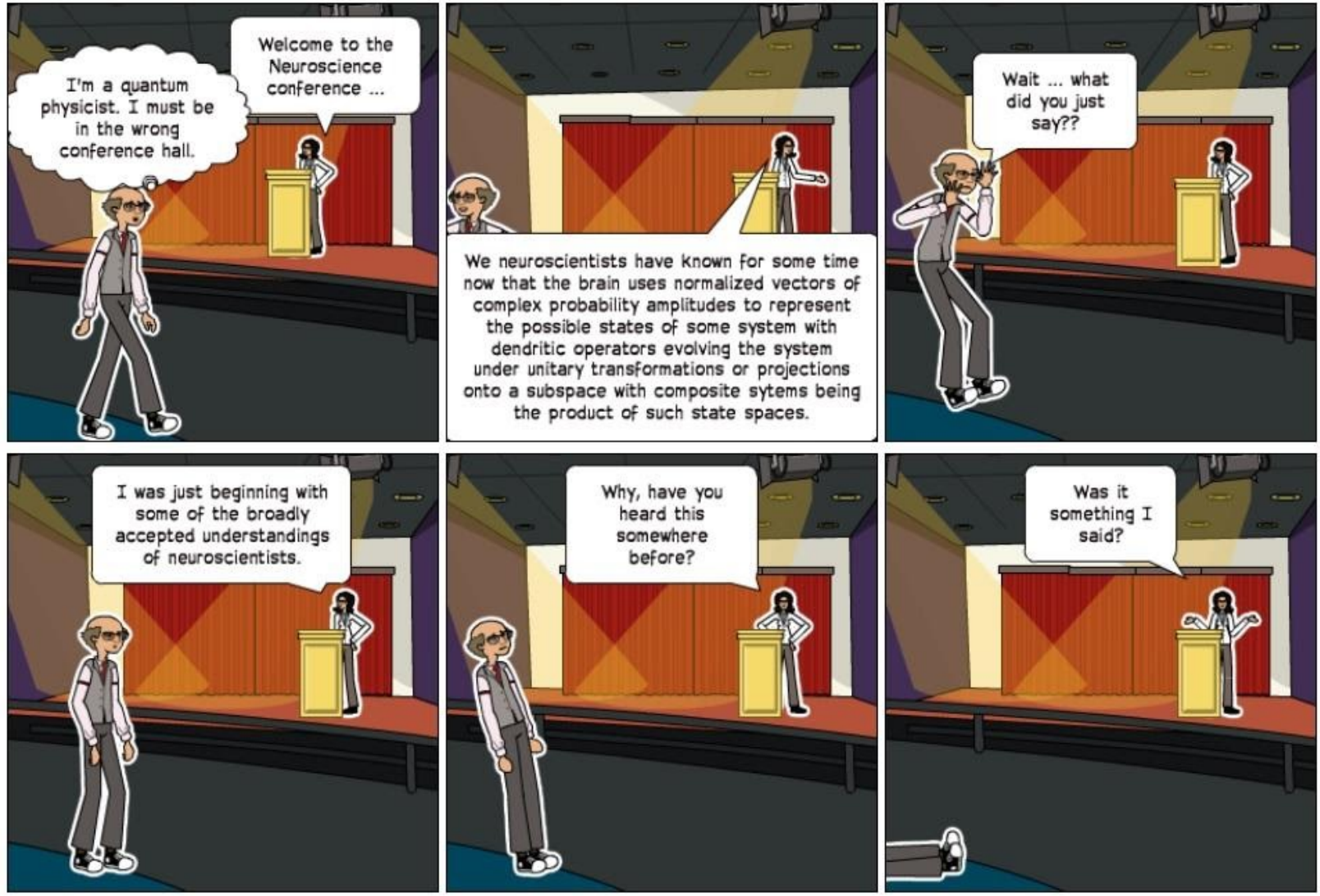


\section{References}

Aaronson S. Is quantum mechanics an island in theoryspace? arXiv:quant-ph/0401062v2. 2004.

Aaronson S. Lecture 9 of PHYS771 Quantum Computing Since Democritus. 2006a.

Aaronson S. Lecture 9 of PHYS771 Quantum Computing Since Democritus. 2006b.

Aaronson S. The learnability of quantum states. arXiv.org/quant-ph/0608142v3. 2007.

Aaronson S. BQP and the polynomial hierarchy. arXiv:0910.4698v1 . 2009.

Aaronson S. Quantum Computing Since Democritus. Cambridge, 2013.

Aaronson S. The power of the Digi-Comp II. 2014. https://www.scottaaronson.com/blog/?p=1902.

Aaronson S. From shtetl to Forum. 2020. https://www.scottaaronson.com/blog/?p=4536

Abbott LF and Blum KI. Functional significance of long-term potentiation for sequence learning and prediction. Cerebral Cortex 1996; 6:406-416.

Abellán C, et al. Challenging local realism with human choices. Nature, 2018; 557, 212-216.

Abulof U. "I see you (The Mind's I\&I)". Sapienism 2019; https://www.sapienism.com/post/_icu.

Adams RA, Shipp S, and Friston KJ. Predictions not commands: active inference in the motor system. Brain Struct Funct 2013; May 218(3):611-43.

Adleman L, Demarrais J, and Huang MA. Quantum computability. SIAM J Comp 1997; 26(5):1524-1540.

Aerts D. Quantum interference and superposition in cognition: Development of a theory for the disjunction of concepts. arXiv:0705.0975v2. 2007.

Aerts D. Quantum structure in cognition. arXiv:0805.3850v2. 2009.

Aerts D, Broekaert J, and Gabora L. A case for applying an abstracted quantum formalism to cognition. New Ideas in Psychology 2011; 29:136-146.

Agmon-Snir H. and Segev I. Signal delay and input synchronization in passive dendritic structures. J Neurophysiology 1993; 70:2066-2085.

Aizenberg I. Complex-Valued Neural Networks with Multi-Valued Neurons. Springer, 2011.

Albert D. Quantum Mechanics and Experience. Harvard, 1992. 
Alimomeni M, Safavi-Naini and Sharifian S. A true random generator using human gameplay. In: Das S.K., Nita-Rotaru C., Kantarcioglu M. (eds) Decision and Game Theory for Security. GameSec 2013. Lecture Notes in Computer Science, vol 8252. Springer, Cham.

Anisomovas E, Gerbier F, Andrijauskas T and Goldman N. Design of laser-coupled honeycomb optical lattices supporting Chern insulators. arXiv:1401.1718v2. 2014.

Arbib M. ed. The Handbook of Brain Theory and Neural Networks. MIT, 1995.

Arkani-Hamed N and Trnka J. The Amplituhedron. Journal of High Energy Physics 2014; 10.

Avitable D. and Wedgwood KCA. Macroscopic coherent structures in a stochastic neural network: from interface dynamics to coarse-grained bifurcation analysis J. Math. Biol. 2017; 75: 885.

Baez JC. The octonions. Bulletin Amer Math Soc 2002; 39:145-205.

Baez JC and Stay M. Physics, Topology, Logic and Computation: A Rosetta Stone. arXiv:0903.0340v3. 2009.

Barlow HB. Single units and sensation: A neuron doctrine for perceptual psychology? Perception 1972; 1:371-394.

Barnum H, Müller MP, and Ududec C, Higher-order interference and single-system postulates characterizing quantum theory. New Journal of Physics, 2014. 16(12):123029.

Barrett JA. The Quantum Mechanics of Minds and Worlds. Oxford, 1999.

Barry C, Hayman R, Burgers N, and Jeffery K. Experience-dependent rescaling of entorhinal grids Nat. Neurosci 2007, 10:682-4.

Bartos M, Vida I. and Jonas P. Synaptic mechanisms of synchronized gamma oscillations in inhibitory interneuron networks. Nat Rev Neurosci, 2007. 8, 45-56.

Basieva I, Cervantes V, Dzhafarov E and Khrennikov K. True contextuality beats direct influences in human decision making. arXiv:1807.05684v3. 2019.

Behera L, Kar I, and Elitzur AC. Recurrent quantum neural network model to describe eye tracking of moving target. Foundations of Physics Letters 2005; 18(4):357-370.

Ben-Yishai R, Bar-Or RL, and Sompolinsky H. Theory of orientation tuning in the visual cortex. Proc Nat Acad Sci USA 1995; 92:3844-3848.

Bernander Ö, Douglas R, Martin KAC and Koch C. Synaptic background activity influences spatiotemporal integration in single pyramidal cells. Proc Natl Acad Sci USA 1991; 88:11569-11573.

Bernander Ö. Synaptic integration and its control in neocortical pyramidal cells. PhD Thesis, California Institute of Technology, 1993. 
Bernander Ö, Douglas RJ, and Koch C. Amplification and linearization of synaptic input to the apical dendrites of cortical pyramidal neurons. J Neurophysiol 1994; 72:2743-2753.

Bernstein E and Vazirani U. Quantum complexity theory. SIAM J Comp 1997; 26(5):1411-1473.

Berthier M and Provenzi E. When geometry meets psycho-physics and quantum mechanics : Modern perspectives on the space of perceived colors. 4th conference on Geometric Science of Information, Aug 2019, Toulouse, France. hal-02145070v2f

Bisio A. The importance of being spectral. Quantum Views 2019: 3,15.

Blutner R and Hochnadel E. Two cubits for C.G. Jung's theory of personality. Cognitive Systems Research 2010; 11(3):243-259.

Bloomfield S. Arithmetical operations performed by nerve cells. Brain Res 1974; 69:115-124.

Box, G. Robustness in the strategy of scientific model building, in Launer, RL; Wilkinson, GN (eds.), Robustness in Statistics, Academic Press, 1979, pp. 201-236.

Boykin PO et al. On universal and fault-tolerant quantum computation. arXiv:quant-ph/9906054. 1999.

Braitenburg V and Schüz A. Cortex: Statistics and Geometry of Neuronal Connectivity, $2^{\text {nd }}$ Ed. Springer-Verlag, 1998.

Branco $\mathrm{T}$ and Hausser M. The single dendritic branch as a fundamental functional unit in the nervous system. Curr Op Neurobio 2010; 20:494-502.

Broekaert JB, Busemeyer JR and Pothos EM. The Disjunction Effect in two-stage simulated gambles. An experimental study and comparison of a heuristic logistic, Markov and quantum-like model.

Cognitive Psychology, 2019, Vol: 117, Page: 101262.

Bruck J. Harmonic analysis of polynomial threshold functions. SIAM J Disc Math 1990; 3:168-177.

Bruck J and Smolensky R. Polynomial threshold functions. SIAM J Comput 1992; 21:33-42.

Brunel N. Dynamics of sparsely connected networks of excitatory and inhibitory spiking neurons. J Comp Neurosci 2000; 8(3):183-208.

Bub J. Quantum mechanics is about quantum information. arXiv:quant-ph/0408020v2. 2008.

Bub J. Bananaworld: Quantum Mechanics for Primates. Oxford, 2016.

Bub J. ‘Two Dogmas’ Redux. arXiv:1907.06240v1. 2019.

Burgess N, Barry C, and O'Keefe J. An oscillatory interference model of grid cell firing. Hippocampus 2007; 17(9):801-812.

Burgess N. Grid cells and theta as oscillatory interference: Theory and predictions. Hippocampus 2008; 18(12):1157-1174. 
Busemeyer JR and Bruza PD. Quantum Models of Cognition and Decision. Cambridge, 2012.

Busemeyer J and Wang Z. Hilbert space multi-dimensional modeling. arXiv:1704.04623v1. 2017.

Busemeyer JR, Kvam PD and Pleskac TJ. Comparison of Markov versus quantum dynamical models of human decision making. doi:10.1002/wcs.1526. 2020.

Bush D and Burgess N. A hybrid oscillatory interference/continuous attractor network model of grid cell firing. J. of Neuroscience 2014; 34(14):5065-5079.

Buxhoeveden DP, and Casanova MF. The minicolumn and evolution of the brain. Brain Behav Evol 2002a; 60:125-151.

Buxhoeveden DP, and Casanova MF. The minicolumn hypothesis in neuroscience. Brain 2002b; 125(5):935-951.

Cain N, Iyer R, Koch C, Mihalas S. The computational properties of a simplified cortical column model. PLoS Comput Biol 2016. 12(9): e1005045.

Cao B and Abbott LF. A new computational method for cable theory problems. Biophys J 1993; 64:303-313.

Carandini M and Heeger DJ. Summation and division by neurons in primate visual cortex. Science 1994; 264:1333-1336.

Carandini M and Heeger DJ. Normalization as a canonical neural computation. Nat Rev Neurosci 2011; Nov $23 ; 13(1): 51-62$.

Carroll S, Josić K. and Kilpatrick Z.P. Encoding certainty in bump attractors. J Comput Neurosci 2014; 37: 29 .

Cervantes V and Ehtibar D. True contextuality in a psychophysical experiment. arXiv:1812.00105v2. 2019.

Chauhan AS, Taylor JD and Nogaret A. Dual mechanism for the emergence of synchronization in inhibitory neural networks. Sci Rep 2018; 8, 11431.

Cheon T and Takahashi T. Interference and inequality in quantum decisions theory. arXiv:1008.2628v2. 2010.

Chiribella G et al. Informational derivation of quantum theory. Phys Rev A 2011; 84

Cinti F. Incommensurability effects on dipolar bosons in optical lattices. arXiv:1510.08734v1. 2015.

Cisek P. Cortical mechanisms of action selection: the affordance competition hypothesis. Philos Trans $\mathrm{R}$ Soc Lond B Biol Sci 2007; 362(1485):1585-99. 
Conte $\mathrm{E}$ et al. Mental states follow quantum mechanics during perception and cognition of ambiguous figures. Open Sys And Info Dyn 2009; 16:1-17.

Conte E. A reformulation of Von Neumann's postulates on quantum measurement by using two theorems in Clifford algebra. Int J Theor Phys 2010; 49:587-614.

Contreras D. Electrophysiological classes of neocortical neurons, Neural Netw. 2004; 17(5-6):633-46.

Conway JH and Kochen S. The Free Will Theorem. Found Phys 2006 36: 1441-1473.

Conway JH and Kochen S. The strong free will theorem. N Amer Math Soc 2009 56: 226-232.

Davis MD. The Undecidable. Raven Press, 1965.

DeBarros JA and Suppes P. Quantum mechanics, interference, and the brain. Journal of Mathematical Psychology 2009; 53:306-313.

Defelipe, J. The neocortical column. Frontiers in Neuroanatomy. 26 June, 2012.

Derrida J. Of Grammatology. Johns Hopkins University Press,1967/1998.

Deutsch D. Quantum theory, the Church-Turing principle and the universal quantum computer. Proc. Roy. Soc. London Ser. A, 400, pp. 97-117. 1985.

Deutsch D. The Beginning of Infinity. Viking, 2011.

Dirac PAM. The Principles of Quantum Mechanics. Oxford, 1930/1958.

DiVincenzo DP. Two-bit gates are universal for quantum computation. Phys Rev A 1995; 51(2):1015-1022.

DiVincenzo DP. Quantum gates and circuits. in Proc ITP Conf on Quantum Coherence and Decoherence. arXiv:quant-ph/9705009 1998. Also Proc R Soc London A 1998; 454:261-276.

DiVincenzo DP. The physical implementation of quantum computation. arXiv:quant-ph/0002077. 2000.

Douglas RJ and Martin KA. Opening the grey box. Trends Neurosci 1991; 14:735-769.

Douglas RJ et al. Recurrent excitation in neocortical circuits. Science 1995; 269:981-985.

Douglas RJ and Martin KAC. Neuronal circuits of the neocortex. Ann Rev Neurosci 2004; 27:419-451.

Douglas RJ and Martin KAC. Recurrent neuronal circuits in the neocortex. Curr Bio 2007; 17(13)496-500.

Dunn B, Mørreaunet M and Roudi Y. Correlations and functional connections in a population of grid cells, arXiv:1405.0044v2. 2014. 
Ebert $\mathbf{J}$ and Wegner D. Mistaking randomness for free will. Consciousness and Cognition 2011. 20(3): 965-71.

Einstein A. Related by Heisenberg, quoted in Unification of Fundamental Forces, Salam A. 1926/1990.

Einstein A in a 1948 letter to Heitler W. In Fine, A. Einstein's Interpretation of Quantum Theory. In Beller M, Cohen RS, Renn J, eds. Einstein in Context, Cambridge, 1993.

Euclid. Elements, 300BC.

Everett H. On the Foundations of Quantum Mechanics. PhD Thesis. Princeton, 1957a.

Everett H. "Relative State" formulation of quantum mechanics, Rev Mod Phys 1957b. 29:454-62

Everett $\mathrm{H}$. The theory of the universal wave function, in DeWitt and Graham, The Many-Worlds Interpretation of Quantum Mechanics. Princeton, 1973.

Fell L, Dehdashti S, Bruza P, and Moreira C. An experimental protocol to derive and validate a quantum model of decision-making, arXiv:1908.07935, 2019.

Feynman RP and Hibbs AR. Quantum Mechanics and Path Integrals. McGraw-Hill, 1965a.

Feynman RP, Leighton RB and Sands M. Lectures on Physics: Probability. Volume I. Chapter VI. Addison-Wesley, 1965b.

Feynman RP. What is science? The Physics Teacher 1969, volume 7, issue 6, p. 313-320.

Feynman RP. Simulating physics with computers. Int J Theor Phys 1982; 21: 467.

Feynman RP. Cargo Cult Science. Caltech Commencement Address 1974.

Feynman RP. Negative probability. In Peat, F. David; Hiley, Basil (eds.). Quantum Implications: Essays in Honour of David Bohm. Routledge \& Kegan Paul Ltd. pp. 235-248. 1987.

Friedberg R and Lee TD. Derivation of Regge's action from Einstein's theory of General Relativity. Nucl. Phys. B 1984; 242, 145-166.

Fields $\mathrm{C}$ and Marciano A. Hierarchical Markov blankets and adaptive active inference: Comment on "Answering Schrödinger's question: A free-energy formulation" by Maxwell James Désormeau Ramstead et al. Phys Life Rev. 2018 Mar;24:27-28.

Frauchiger, D., Renner, R. Quantum theory cannot consistently describe the use of itself. Nat Commun 2018; 9, 3711

Fries P, Nikoli D and Singer W. The gamma cycle. Trends in Neurosci 2007; 50(7):309-316.

Fries P. Neural gamma-band synchronization as a fundamental process in cortical computation. Ann Rev Neurosci 2009; 32:209-224. 
Friston K. Functional Connectivity: Eigenimages and multivariate analyses. Chapter 49 of Human Brain Function, Academic Press, 2004. https://www.fil.ion.ucl.ac.uk/spm/doc/books/hbf1/Ch5.pdf

Fromherz P and Gaede V. Exclusive-OR function of single arborized neuron. Bio Cybern 1993; 69:337-344.

Fuchs CA. QBism the perimeter of quantum Bayesianism. arXiv:quant-ph/1003.5209.v1. 2010.

Fung CCA, Wong KYM and Wu S. A moving bump in a continuous manifold: A comprehensive study of the tracking dynamics of continuous attractor neural networks. arXiv:0808.2341v3. 2009.

Fung CCA, Wong KYM and Wu S. Dynamics of neural networks with continuous attractors. arXiv:0801.4461v2. 2015.

Gabbiani F and Koch C. Principles of spike train analysis. In: Koch and Segev, eds, Methods in Neuronal Modeling, 313-360. MIT, 1998.

Gell-Mann M and Hartle JB. Quantum mechanics in the light of quantum cosmology, in Zurek, Complexity, Entropy, and the Physics of Information 8:425-58.

Gestri G. Pulse frequency modulation in neural systems: A random model. Biophys 1971; 11:98-109.

Girard J-Y. The Blind Spot: Lectures on Logic. European Mathematical Society. 2011.

Gisin N. Quantum Chance: Non locality, Teleportation and Other Quantum Marvels. Springer, 2014.

Giovanneti V, Lloyd S, and Maccone L. Quantum-enhanced measurements: beating the standard quantum limit. Science 2004; 306:1330.

Gleason AM. Measures on the closed subspaces of a Hilbert space. J Math Mech 1957; 6:885-893.

Gödel K. Collected Works, Volume III. Oxford, 1961/1981.

Grover LK. Quantum mechanics helps in searching for a needle in a haystack. Phys Rev Lett 1997; 79(2):325.

Gunji Y-P, Nishiyama Y, and Adamatzky A. Robust soldier crab ball gate. arXiv:1204.1749v1. 2012.

Grgurich R and Blair HT. An uncertainty principle for neural coding: Conjugate representations of position and velocity are mapped onto firing rates and co-firing rates of neural spike trains. Hippocampus. 2020;30:396-421.

Gudder S. Quantum Probability. Academic Press, 1988.

Gudder S. Quantum Markov chains. J Math Phys 2008; 49(7).

Halmos PR. Finite-Dimensional Vector Spaces. Van Nostrand, 1958; also Springer, 1993. 
Halprin R and Naor M. Games for extracting randomness. Symposium on Usable Privacy and Security (SOUPS) 2009, July 15-17.

Harrow A. Coherent communication of classical messages. Phys Rev Lett 2004; 92(9):097902.

Hasselmo $\mathrm{M}$ and Brandon $\mathrm{M}$. A model combining oscillations and attractor dynamics for generation of grid cell firing. Frontiers in Neural Circuits 2012; 6:1-13.

Hawking SW and Ellis GFR. The Large Scale Structure of Space-Time. Cambridge, 1973.

Hebb DO. The Organization of Behavior. John Wiley, 1949.

Hines M. Effective computation of branched nerve equations. Intl J Bio Comp 1984; 15:69-76.

Horn RA and Johnson CR. Matrix Analysis. Cambridge, 1985.

Horton JC and Adams DL. The cortical column: a structure without a function. Philos Trans R Soc Lond B Biol Sci 2005; 360(1456):837-62.

Hosoya T. The basic repeating modules of the cerebral cortical circuit. Proc Jpn Acad Ser B Phys Biol Sci. 2019;95(7):303-311.

Innocenti GM and Vercelli A. Dendritic bundles, minicolumns, columns, and cortical output units. Front. Neuroanat, 2010. 4:11.

Jaeger CB and Trueblood J. Thinking Quantum: A new perspective on decision making in law. Florida State University Law Review 2019; 46(4):733.

Janas M, Cuffaro M and Janssen M. Putting probabilities first: How Hilbert space generates and constrains them. arXiv:1910.10688v1. 2019.

Jaynes ET. Gibbs vs Boltzmann entropies. American Journal of Physics 1965; 33(5):391-398.

Jencova A and Plavala M. On the properties of spectral effect algebras. arXiv:1811.12407v2. 2018.

Jozsa R. Entanglement and quantum computation, in Foundations of Science. Hugget et al., eds. Geometric Issues Oxford, 1997.

Kant, I. Critique of Pure Reason. Cambridge University Press. 1781/1998.

Kastner, RE. The illusory appeal of decoherence in the Everettian picture: Affirming the consequent. arXiv:1603.04845v1. 2016.

Keane A and Gong P. Propagating waves can explain irregular neural dynamics. Journal of Neuroscience 2015, 35 (4) 1591-1605.

Keller N and Klein O. Quantum speedups need structure. arXiv:1911.03748v1. 2019. 
Kempe J et al. Entangled games are hard to approximate. FOCS '08 Proceedings of the 2008 49th Annual IEEE Symposium on Foundations of Computer Science 447-456. 2008.

Khrennikov A. A model of quantum-like decision making with applications to psychology and cognitive science. Biosystems 2009; 95:179-187.

Killoran N, Bromley TR, Arrazola JM, Schuld M, Quesada N and Lloyd S. Continuous-variable quantum neural networks. Phys. Rev. Research 2019; 1, 033063.

Kilpatrick Z and Ermentrout B. Wandering bumps in stochastic neural fields. arXiv:1205.3072v1. 2012.

Klausberger T and Somogyi P. Neuronal diversity and temporal dynamics : The unity of hippocampal circuit operations. Science 2008; 321:53-57.

Knight B. Dynamics of encoding in a population of neurons. J Gen Physiol 1972; 59:734-766.

Koch C and Poggio T. Multiplying with synapses and neurons. In: McKenna et al., eds, Single Neuron Computation, 315-345. Academic Press, 1992.

Koch C. Biophysics of Computation. Oxford, 1999.

Koch C and Hepp K. Quantum mechanics in the brain. Nature, 2006; 440 (7084). p. 611.

Kochen S and Specker EP. On the problem of hidden variables in quantum mechanics. J Math and Mech 1967 17: 59-87.

Kraus K. States, Effects, and Operations: Fundamental Notions of Quantum Theory. Lecture Notes in Physics, vol. 190. Springer-Verlag, 1983.

Le Penne, S. "What Do You See?". Sapienism 2019; https://www.sapienism.com/post/what-do-you-see.

Landsman K. Randomness? What randomness? arXiv:1908.07068v2. 2019.

Llinás P. Tensor network theory of the metaorganization of functional geometries in the CNS. Neuroscience $1985 ; 16: 245-273$.

Lloyd S. The computational universe: Quantum gravity from quantum computation. arXiv:0501135v8. 2006.

Lloyd S. A Turing test for free will. arXiv:1310.3225. 2013a.

Lloyd S. Uncomputability and physical law. arXiv:1312.4456v1. $2013 b$.

London M and Segev I. Conducting synaptic music in dendrites. Nature Neuro 2004; 7(9):4-5.

Lubenov EV and Siapas AG. Hippocampal theta oscillations are traveling waves. Nature. 2009 May 28;459(7246):534-9. 
Lundqvist $\mathrm{M}$ et al. Attractor dynamics in a modular network model of neocortex. Network: Computation in Neural Systems 2006; 1-24.

Lundqvist M, Compte A and Lansner A. Bistable, irregular firing and population oscillations in a modular attractor memory network. PLoS Comput Biol 2010; 6(6):e1000803.

Masanes, L., Galley, T.D. \& Müller, M.P. The measurement postulates of quantum mechanics are operationally redundant. Nat Commun 2019; 10, 1361.

Masuyama N and Loo CK. Quantum-inspired complex-valued multidirectional associative memory. 2015 International Joint Conference on Neural Networks (IJCNN).

Maudlin T. Quantum Non-Locality and Relativity. Wiley-Blackwell, 2011.

Mel BW. NMDA-based pattern discrimination in a modeled cortical neuron. Neural Comput 1992; 4:502-517.

Mel BW. Synaptic integration in an excitable dendritic tree. J Neurophysiol 1993; 70:1086-1101.

Mel BW. Information processing in dendritic trees. Neural Comput 1994; 6:1031-1085.

Mel BW et al. Translation-invariant orientation-tuning in visual complex cells could derive from intradendritic computations. J Neurosci 1998; 18:4325-4334.

Merker B. Consciousness without a cerebral cortex: A challenge for neuroscience and medicine. Behav Brain Sci 2007; 30(1):63-81.

Miles R. Diversity in inhibition. Science 2000; 287:244-246.

Miller WA. The Hilbert action in Regge calculus. Class. Quantum Grav 1997;14 L199-L204 (14); arXiv:gr-qc/9708011.

Molnar $\mathrm{G}$ et al. Complex events initiated by individual spikes in the human cerebral cortex. PLoS Biol 2008; 6(9):3222.

Montague PR and Sejnowski TJ. The predictive brain: Temporal coincidence and temporal order in synaptic learning mechanisms. Learning and Memory 1994; 1:1-33.

Moore C and Mertens S. The Nature of Computation. Oxford, 2011.

Moreira C et al. Towards a quantum-like cognitive architecture for decision making. arXiv:1905.05176v1. 2019.

Moser E and Moser M. A metric for space. Hippocampus 2008; 18:1142-1156.

Moser Group, https://www.ntnu.edu/kavli/discovering-grid-cells, 2019.

Mountcastle VB. The columnar organization of the neocortex. Brain 1997; 120(4):701-22. 
Młynarski W. Sparse, complex-valued representations of natural sounds learned with phase and amplitude continuity priors. arXiv:1312.4695v3. 2014.

Młynarski W. The Opponent Channel Population Code of Sound Location Is an Efficient Representation of Natural Binaural Sounds. PLoS Comput Biol 2015; 11(5): e1004294.

Muller L, Chavane F, Reynolds J, Sejnowski TJ. Cortical travelling waves: mechanisms and computational principles. Nat Rev Neurosci. 2018 May;19(5):255-268.

Nelson D and McEvoy C. Entangled structures and context. In: Bruza et al., eds, Proc of the AAAI Spring Symposium on Quantum Interaction. AAAI Press, 2007.

Nielsen M and Chuang I. Quantum Computation and Quantum Information. Cambridge, 2000.

Niestegge G. Local tomography and the role of the complex numbers in quantum mechanics. arXiv:2001.11421v1.

Nunez R and Cooperrider K. The tangle of space and time in human cognition. Trends in Cog. Sci 2013; 17(5)220-229.

O'Keefe J and Burgess N. Dual phase and rate coding in hippocampal place cells: theoretical significance and relationship to entorhinal grid cells. Hippocampus 2005; 15(7):853-866.

Palais R. S. "The Classification of Real Division Algebras" American Mathematical Monthly 75:366-8.

Papoutsi A, Kastellakis G, Psarrou M, Anastasakis S, Poirazi P. Coding and decoding with dendrites. J Physiol Paris. 2014 Feb;108(1):18-27.

Pastoll $\mathrm{H}$ et al. Feedback inhibition enables theta-nested gamma oscillations and grid firing fields. Neuron $2013 ; 77: 141-154$.

Pavičić M. Classical logic and quantum logic with multiple and common lattice models 2016; Advances in Mathematical Physics, vol. 2016, Article ID 6830685.

Pearl J. Probabilistic Reasoning in Intelligent Systems: Networks of Plausible Inference. Morgan Kaufmann. 1988.

Pearlmutter BA and Zador A. Sparse matrix methods for modeling single neurons. In: Koch, C. Biophysics of Computation 1999; 487-502.

Penrose R. The Road to Reality. BCA, 2004.

Peres A. Quantum Theory: Concepts and Methods. Kluwer Academic, 1993.

Pessoa JSO. How can the decoherence approach help to solve the measurement problem? Synthese 1998; 113:323.

Pitowsky I. Quantum mechanics as a theory of probability. arXiv:quant-ph/0510095v1. 2008a. 
Pitowsky I. Geometry of quantum correlations. Phy Rev A 2008b; 77:62109.

Pothos E, Busemeyer J, Shiffrin R and Yearsley J. The rational status of quantum cognition. J Exp Psychol Gen. 2017 Jul;146(7):968-987

Qin Y, Kawano Y, Portoles O and Cao M. Partial phase cohesiveness in networks of Kuramoto oscillator networks. arXiv:1906.01065v1. 2019.

Quiroga RQ. Concept cells: the building blocks of declarative memory functions. Nat Rev Neurosci 2012; 13(8):587-597.

Rakic P. Confusing cortical columns. PNAS 2008; 105(34):12099-12100.

Rall W and Rinzel J. Branch input resistance and steady attenuation for input to one branch of a dendritic neuron model. Biophys J 1973; 13:648-688.

Rapoport A and Budescu D. Generation of random series in two-person strictly competitive games. Journal of Experimental Psychology: General 1992. 121, 3, 352-363.

Regge T. General Relativity without Coordinates. Nuovo Cimento 1961; 19, 558-571 1961

Reichardt W. Autocorrelation, a principle for evaluation of sensory information by the central nervous system. In: Rosenblith, ed, Principles of Sensory Communication, 303-317. Wiley, 1961.

Rieke E et al. Spikes: Exploring the Neural Code. MIT, 1997.

Rorty R. Contingency, Irony, and Solidarity. Cambridge, 1989.

Rowland DC, Roudi Y, Moser M-B and Moser E. Ten Years of Grid Cells. Annual Review of Neuroscience 2016 39:1, 19-40.

Royer A. Reduced dynamics with initial correlations, and time-dependent environment and Hamiltonians. Phys Rev Lett 1996; 77(16):3272-3275.

Rudin W. Functional Analysis, Tata MacGraw-Hill, 1973.

Sainz AB, Guryanova Y, Acin A and Navascues M. Almost quantum correlations violate the norestrictions hypothesis. arXiv:1707.02620v3. 2018.

Sarovar M et al. Quantum entanglement in photosynthetic light-harvesting complexes. Nature Physics 2010; 6:462-67.

Schmid D, Selby JH, Wolfe e, Kunjwal R and Spekkens RW. The characterization of noncontextuality in the framework of generalized probabilistic theories. arXiv:1911.10386v1. 2019.

Schlosshauer M, Kofler J and Zeilinger A. A snapshot of foundational attitudes towards quantum mechanics. arXiv:1301.1069v1. 2013. 
Segev I. What do dendrites and their synapses tell the neuron? J Neurophys 2006; 95:1295-1297.

Sharifian S. Random Number Generation using Human Gameplay (Unpublished master's thesis) 2016. University of Calgary, Calgary, AB.

Shipp S. Structure and function of the cerebral cortex. Curr Biol 2007; 17:1-6.

Shrapnel S, Costa F and Milburn G. Updating the Born rule. arXiv:1702.01845v1. 2017.

Simon DR. On the power of quantum computation. SIAM J Comput 1997; 26(5):1474-1483.

Sokal A. Introduction to normed linear spaces. 2013. Lecture Notes in Functional Analysis. https://www.ucl.ac.uk/ ucahad0/3103 handout 3.pdf

Srinivasan MV and Bernard GD. A proposed mechanism for multiplication of neural signals. Biol Cybern $1976 ; 21: 227-236$.

Stewart I. Why Beauty is Truth. Basic Books, 2008.

Strang G. Linear Algebra and its Applications. Academic Press, 1980.

Suarez H and Koch C. Linking linear threshold units with quadratic models of motion perception. Neural Comp 1989; 1:318-320.

Sun W and Dan Y. Layer-specific network oscillation and spatiotemporal receptive field in the visual cortex. Proc Nat Acad Sci USA 2009; 106(42):17986-17991.

Suppes $\mathrm{P}$ et al. Phase-oscillator computations as neural models of stimulus-response conditioning and response selection. Journal of Mathematical Psychology 2012; 56(2):95-117.

Svozil K. Physical (A)Causality: Determinism Randomness and Uncaused Events, Springer, 2018.

Takeda M, Hirabayashi T, Adachi Y and Miyashita Y. Dynamic laminar rerouting of inter-areal mnemonic signal by cognitive operations in primate temporal cortex. Nature Communications 2018; 9:4629.

Tam D. A spike train analysis for quantifying inhibitory near synchrony in spike firings. Neurocomputing 2002; 1149-1153.

Tanaka $\mathrm{H}$ and Nelson DR. Non-Hermitian quasi-localization and ring attractor neural networks. arXiv:1811.07433v1. 2018.

Tegmark M. The importance of quantum decoherence in brain processes. arXiv:9907009v2.

Thompson AM et al. Synaptic connections and small circuits involving excitatory and inhibitory neurons in layers 2-5 of adult rat and cat neocortex: triple intracellular recordings and biocytin labeling in vitro. Cereb Cortex 2002; 12(9):936-53

Torre V and Poggio T. A synaptic mechanism possibly underlying directional selectivity to motion. Proc Roy Soc Lond B 1978; 202:409-416. 
Trueblood J, Yearsley J and Pothos E. A quantum probability framework for human probabilistic inference. J Exp Psychol Gen. 2017 Sep;146(9):1307-1341.

Tsunoda $\mathrm{K}$ et al. Complex objects are represented in macaque inferotemporal cortex by the combination of feature columns. Nat Neurosci 2001; 4(8):832-8.

Turing AM. The chemical basis of morphogenesis. Philos. Trans. R. Soc. 1952. 237:37-72.

Uprety S, Tiwari P, Dehdashti S, Fell L, Song D, Bruza P and Melucci M. Quantum-like structure in multidimensional relevance judgements. arXiv:2001.07075v1.

Vainstein $\mathrm{F}$ and Jones $\mathrm{C}$. Checking computation of numerical functions by the use of functional equations. Reliable Engineering Computing, 2006.

Vazirani U and Vidick T. Certifiable quantum dice - Or, testable exponential randomness expansion. arXiv:1111.6054v1. 2011.

Vidotto F. On the tensor product structure of general covariant systems. Quantum Foundations 2020. http://pirsa.org/20030072

Vitiello G. Fractals and the Fock-Bargman representation of coherent states. In: Bruza et al., eds, QI2009 Lecture Notes in Artificial Intelligence, 5494:6-16. Springer, 2009.

Von Neumann J. Mathematical Foundations of Quantum Theory. Princeton, 1932/1955.

Von Neumann J. Probabilistic Logics and Synthesis of Reliable Organisms from Unreliable Components. In Automata Studies, eds. Shannon C and McCarthy J, pp. 43-98, Princeton University Press. 1956.

Von Neumann J. Quantum logics (strict- and probability-logics). In Collected works, vol. IV, pp. 195-197. Oxford, UK: Pergamon Press. 1962.

Wald RM. General Relativity. Chicago, 1984.

Wang H, Lam K, Fung CCA, Wong KYM and Wu S. A rich spectrum of neural field dynamics in the presence of short-term synaptic depression. arXiv:1502.03662v1. 2015.

Ward LM. Synchronous neural oscillations and cognitive processes. Trends in Cog Sci 2003; 7(12):553-559.

Watrous J. PSPACE has 2-round quantum interactive proof systems. arXiv:cs/9901015. 1999.

Wei X, Prentice J and Balasubramanian V, The Sense of Place: Grid Cells in the brain and the Transcendental number e. arXiv:1304.0031. 2013.

Wiesner S. Conjugate Coding. SIGACT News 1983; 15 (1): 78-88. 
Wigner E. The unreasonable effectiveness of mathematics in the natural sciences, Communications Pure and Applied Mathematics 1960; 13(1).

Witten E. Magic, mystery and matrix. Notices of American Mathematical Society 1998; 45:1124-1129.

Wittgenstein L. Tractatus Logico Philosophicus. Dover, 1921.

Wittgenstein L. Philosophical Investigations. Blackwell Publishing, 1953/2001.

Wojciechowski BW and Pothos EM. Is There a Conjunction Fallacy in Legal Probabilistic Decision Making? Front. Psychol. 2018; 9:391.

Wu S, Wong KY, Fung CC, Mi Y, Zhang W. Continuous Attractor Neural Networks: Candidate of a Canonical Model for Neural Information Representation. F1000Res. 2016;5:F1000 Faculty Rev-156. Published 2016 Feb 10. doi:10.12688/f1000research.7387.1

Yao AC. Quantum circuit complexity. Proc of the $34^{\text {th }}$ Ann IEEE Symp On Found of Comp Sci 1993; 352-361.

Yearsley $\mathrm{J}$ and Halliwell $\mathrm{J}$. Contextuality in human decision making in the presence of direct influences: A comment on Basieva et al. (2019). arXiv:1905.12570v2. 2019.

Yoon K, Buice MA, Barry C, Hayman R, Burgess N, Fiete IR. Specific evidence of low-dimensional continuous attractor dynamics in grid cells. Nat Neurosci. 2013;16(8):1077-1084.

Zador AM, Agmon-Snir H and Segev I. The morphoelectronic transform: A graphical approach to dendritic function. J Neurosci 1995; 15:1668-1682.

Zador AM and Dobrunz LE. Dynamic synapses in the cortex. Neuron 1997; 19:1-4.

Zanardi P, Lidar D and Lloyd S. Quantum tensor product structures are observable-induced. arXiv:0308043v1.

Zhao M-J and Jaeger H. Norm observable operator models. Neural Computation. 2010 Jul;22(7):1927-59.

Zurek WH. Decoherence and the transition from quantum to classical. Phys Today 1991; Oct.

Zurek WH. Decoherence and the transition from quantum to classical - REVISITED. arXiv:quant-ph/0306072v1. 2003. 
